\title{
A New Threshold Reveals The Uncertainty About The Effect of School Opening On Diffusion of Covid- 19.
}

\author{
Alberto Gandolfi ( $\square$ ag189@nyu.edu ) \\ New York University Abu Dhabi \\ Andrea Aspri \\ University of Pavia \\ Elena Beretta \\ Politecnico di Milano \\ Khola Jamshad \\ New York University Abu Dhabi \\ Muyan Jiang \\ New York University Abu Dhabi
}

\section{Research Article}

Keywords: epidemic, Covid-19, effect of school opening, in-class teaching, remote learning, SIR model, subpopulations, phase transition, vaccines

Posted Date: May 24th, 2021

DOI: https://doi.org/10.21203/rs.3.rs-472359/v1

License: (c) (1) This work is licensed under a Creative Commons Attribution 4.0 International License.

Read Full License

Version of Record: A version of this preprint was published at Scientific Reports on February 22nd, 2022. See the published version at https://doi.org/10.1038/s41598-022-06540-w. 


\title{
A new threshold reveals the uncertainty about the effect of school opening on diffusion of Covid-19.
}

\author{
Alberto Gandolfi ${ }^{1}$, Andrea Aspri ${ }^{2}$, Elena Beretta ${ }^{3}$, Khola \\ Jamshad $^{1}$, and Muyan Jiang ${ }^{1}$ \\ ${ }^{1}$ New York University Abu Dhabi \\ ${ }^{2}$ Università di Pavia \\ ${ }^{3}$ New York University Abu Dhabi and Politecnico di Milano
}

\begin{abstract}
We aim at clarifying the controversy about the effects of school openings or closures on the course of the Covid-19 pandemic.

The mathematical analysis of compartmental models with subpopulations shows that the in-school contact rates affects the overall course of the pandemic only above a certain threshold that separates an influence phase from a non-influence one. The threshold, that we calculate via linear approximation in several cases, seems to appear in all contexts, including outbreaks or new strains upsurge, lockdowns, and vaccination campaigns excluding children, albeit with different values. Our theoretical findings are then confirmed by several data driven studies that have previously identified the phase transition in specific cases.

Specific outcomes of this study are:

- opposite conclusions reached by studies of the same or similar situations might depend on, possibly small, differences in modeling or in parameter estimation from the very noisy Covid-19 data, that result in identifying different phases;

- it is possible to keep schools open at any stage of the Covid19 pandemic, but suitably strict rules must be applied at all times or else this becomes highly detrimental to virus containment efforts;
\end{abstract}

AMS 2020 subject classifications: 37N99

Key words and phrases: epidemic, Covid-19, effect of school opening, in-class teaching, remote learning, SIR model, subpopulations, phase transition, vaccines. 
- as the threshold during vaccination turns out to correspond to the internal transmission rate that would lead to virus extinction if the school population was isolated, the needed strict control can be sustained only for very brief periods; as a result, either schools will have to face a prolonged closure or children need to be vaccinated as well.

\section{Introduction}

This paper stems from the attempt that our group made some months ago to use some of the available data to clarify the potential effects of school opening on the course of the Covid-19 outbreaks. The question of opening or closing schools had already turned out to be one of the most debated issues of the pandemic, affecting more than one billion students, their teachers and communities. Schools had been closed at the early stages of the pandemic in almost every country, with classes held online for most of last year, [14,21]; but the, sometimes hurriedly arranged, remote teaching was creating great difficulties to students, teachers and families $[16,18]$.

As we kept exploring and trying to model data, we ran into a seemingly very unstable situation. Our compartmental models, similar to many others currently used, were getting good approximations, but they relied on parameters, such as duration of clinical symptoms or age dependent susceptibility, previously estimated by other studies. We noticed then that the effect of school opening that the models were asserting depended in a crucial way on small changes in these parameters. We kept oscillating between making the schools the culprits of the pandemic, or completely absolving them, a situation schematically represented in Figures 3-5 below. When we looked at other studies appearing at the time, it seemed that they also offered different, and sometimes opposite views $[3,5,13,15,19,34]$, even with almost the same data.

Our current aim is to propose a reason for such instability, and for the consequent lack of reliability of many studies on this topic. In addition, we would like to offer a theoretical framework on which school opening policies could be based from now on.

Before starting, let's clarify that, in focusing on the effects of in-school transmission on the course of the pandemic, we disregard many other relevant epidemiological issues concerning school opening, such as the possible role and availability of teachers [6]; the sustainability of school opening [11]; as well as psychological, cultural, educational aspects; that need then to be added when planning concrete policies.

On the other hand, keeping models simple in the spirit of $[2,7]$, might help highlighting the fundamental mechanisms at work. We consider, then, an SIR model with two subpopulations and a minimal number of parameters, studying how changes in the transmission rate in one population affect the overall course of the infection. 
The outcome of our analysis has been the existence of a perhaps surprising threshold, below which further reduction of in-school transmission, with closure for instance, has almost no effect, but above which school opening becomes the leading factor driving infections.

We proceeded to test various scenarios: uncontrolled outbreaks or new strains upsurge, which occur at the early stages of virus diffusion and after the release of successful rigid controls; lockdowns; and successful vaccination campaigns on the largest, out-of-school, subpopulation. And we later tested some more elaborate models with pre- and a-symptomatic and many other realistic features. We also review many data based studies from the perspective of this newly discovered phenomenon. The sharp transition showed up in each context, indicating that a general mechanisms is at work.

We proceed to describe the details of the phase transition phenomena, adding rigorous proofs based on the linear approximation of the simplest models. We also compute the explicit values of the thresholds in the various scenarios.

Of particular interest is the case of a vaccination campaign of adults, in which the threshold for in-school transmission rates turns out to be extremely small, giving a strong indication of the need of a children vaccination campaign.

\section{Results}

\subsection{There is a phase transition in the effect of school trans- mission rates on the overall epidemic course.}

In a simple SIR model with subpopulations 1 and 2, in-school and out-of-school, with fractions $S_{1}(0)$ and $S_{2}(0)$ of susceptibles, respectively, and transmission rates $\beta_{i j}$ from population $j$ to population $i$, the effect of $\beta_{11}$ on the course of the epidemic undergoes a phase transition with threshold

$$
\beta_{11}^{*}=\beta_{22} S_{2}(0) / S_{1}(0)
$$

The total number of active cases is almost constant for all $\beta_{11} \in\left[0, \beta_{11}^{*}\right]$, and has a sharp increase for $\beta_{11}>\beta_{11}^{*}$. An effective containment of the effect of a change of $\beta_{11}$ is achieved if

$$
\beta_{11}<\beta_{22} S_{2}(0) / S_{1}(0)-\alpha \sqrt{\beta_{12} \beta_{21} S_{2}(0) / S_{1}(0)},
$$

for some suitable multiple $\alpha$, while there is a substantial effect if

$$
\beta_{11}>\beta_{22} S_{2}(0) / S_{1}(0)+\alpha \sqrt{\beta_{12} \beta_{21} S_{2}(0) / S_{1}(0)} .
$$

Since in concrete cases $\beta_{12}, \beta_{21}<<\beta_{22}[10,20]$ the above values are close to $\beta_{11}^{*}$.

Calculations are done in a linear approximation of the SIR model, which applies to the Covid-19 pandemic as the numbers of active cases are kept relatively low by containment measures in the early stages of the outbreaks [28]. In

the linear approximation it is possible to formally compute the total number of 
cases up to a certain time $\bar{t}$, which corresponds to when a lock down is imposed. If the target is to contain the increase in the number of total cases up to $\bar{t}$ to a given percentage $\epsilon$, an explicit formula allows to compute the maximal allowed value of $\beta_{11}$.

In a realistic example with total population and recovery rate $\gamma$ normalized to 1 , setting $S_{1}(0)=0.2, S_{2}(0) \approx 0.8, \beta_{12}=\beta_{21}=0.5, \beta_{22}=2$, the critical point is $\beta_{11}^{*} \approx 8$. Assuming an initial fraction of $3 \times 10^{-5}$ of active cases in Subpopulation 2 and none in Subpopulation 1, a rescaled time frame of $\hat{t}=5$ (corresponding to approximately 50 days), and $\epsilon=0.3$, a suitable value of $\alpha$ gives $\beta_{11} \leq 6.344$.

The first part of Figure 1 , for $t \in[0,5]$, shows a simulation of the active cases with the above values. One can see that school opening has a moderate effect for small values of $\beta_{11}$, and then the effect becomes dramatic as the values increase past the critical point. More detailed illustrations are in the online material.

It follows that closing schools, i.e. setting $\beta_{11}=0$, is of limited impact if the reproduction rate $\beta_{11} S_{1}(0)$ in school is somewhat lower than $\beta_{22} S_{2}(0)$, the external reproduction rate, and of substantial impact otherwise. This provides harmless school opening options, assuming that one has access to the reproduction rates in the subpopulations.

\subsection{The phase transition is preserved under lock-down, albeit with a different critical point.}

An analogous effect takes place when a lockdown is imposed. If at some time $\bar{t}$ transmission rates are reduced to values $\bar{\beta}_{i j}$, corresponding to a subcritical reproduction number, then the effect of $\bar{\beta}_{11}$ on the total number of active cases undergoes the same phase transition as during the outbreak, but with critical point

$$
\bar{\beta}_{11}^{*}=\frac{1}{S_{1}(\bar{t})}-\frac{\bar{\beta}_{12} \bar{\beta}_{21} S_{1}(\bar{t}) S_{2}(\bar{t})}{S_{1}(\bar{t})\left(1-\bar{\beta}_{22} S_{2}(\bar{t})\right)} .
$$

More precisely, let

$$
A=\frac{\Delta S\left(\bar{\beta}_{11}\right)}{\left(S_{1}(\bar{t})+S_{2}(\bar{t})\right)}=\frac{\left(S_{1}(\infty)-S_{1}(\bar{t})+S_{2}(\infty)-S_{2}(\bar{t})\right)}{\left(S_{1}(\bar{t})+S_{2}(\bar{t})\right)}
$$

indicate the attack rate of the epidemic, i.e. the fraction of the initially susceptible population that is eventually infected by the disease in the course of the epidemic from $\bar{t}$ to complete eradication. It turns out that a sufficient condition to ensure that $\Delta S\left(\bar{\beta}_{11}\right)$ does not exceeds $(1+\epsilon) \Delta S(0)$ is

$$
\bar{\beta}_{11}<F(\epsilon)
$$

where $F$ (see (26) below) is a function that depends on the proportions of active cases and susceptible individuals at time $\bar{t}$. 
In a realistic example continuing the one for the outbreak, with $\bar{\beta}_{12}=\bar{\beta}_{21}=$ $0.25, \bar{\beta}_{22}=1, \epsilon=0.3$, in order to contain the increase in attack rate to no more than $30 \%$ one needs now to have

$$
\bar{\beta}_{11}<2.9944 \text {. }
$$

Although in a different scenario, this is smaller than the value 6.344 found in the outbreak, as there the aim was just to avoid producing an even more extended diffusion of the infection.

The second part of Figure 1, for $t \in[5,18]$, illustrates active cases in the lockdown scenario, with the above values of the model parameters.

When considering a complete outbreak-lockdown cycle, the attack rate undergoes a similar transition, depending on the values of the two transmission rates $\beta_{11}$ and $\bar{\beta}_{11}$. If the pair is sufficiently closed to $(0,0)$, then there is little change in $\Delta(S)$, while there is a drastic change for larger values of the two transmission rates (see Figure 9).

\subsection{Success of widespread vaccination of non-schooling individuals requires internal reproduction number in schools to be subcritical.}

If a vaccination campaign for not-in-school individuals is carried out, the total number of cases from a restart of the epidemic to the complete disappearance due to vaccination undergoes an analogous phase transition, with threshold

$$
\widetilde{\beta}_{11}^{*}=1 / S_{1}(0) .
$$

The attack rate is only moderately changed for $\widetilde{\beta}_{11}$ below the threshold, while the outcome of the vaccination process is substantially disrupted for larger values of $\widetilde{\beta}_{11}$.

Notice that if $\widetilde{\beta}_{11}<\widetilde{\beta}_{11}^{*}$ then the in-school reproduction number $R_{S}=$ $\widetilde{\beta}_{11} S_{1}(0)$ is subcritical, i.e. less than one.

With the data of the previous examples, suppose a vaccination program is introduced targeted to a $60 \%$ coverage in about 3 months (Israel kept this pace at the time we are writing, with schools almost completely closed). Since the internal reproduction number is $R_{S}=0.198872 \widetilde{\beta}_{11}$, then the critical value for $\widetilde{\beta}_{11}$ is 5.02836. This is seen in the third part of Figure 1 , for $t \in[18,31]$. To achieve a sensible containment that limits the number of extra infections to no more than $30 \%$ one needs to take $\widetilde{\beta}_{11}<3.03111$. 


\subsection{From the point of view of containing the epidemic, schools can be kept open at all times, with strict con- trol measures}

Taken together, the results obtained from simple SIR models with subpopulations show that, although the values of the critical points are different, opening of schools would not seriously affect the course of the pandemic at all times, provided the internal transmission rate is kept low enough. On the other hand, if the control is released, then the effect of school opening becomes dramatic.

Figure 1 summarizes the numbers of active cases in the three scenarios we have analyzed: the cyan curve corresponds to closed schools, while the green one is a subcritical pattern; the red curves, instead, show the risk that the pandemic spirals out of control because of insufficiently controlled school opening.

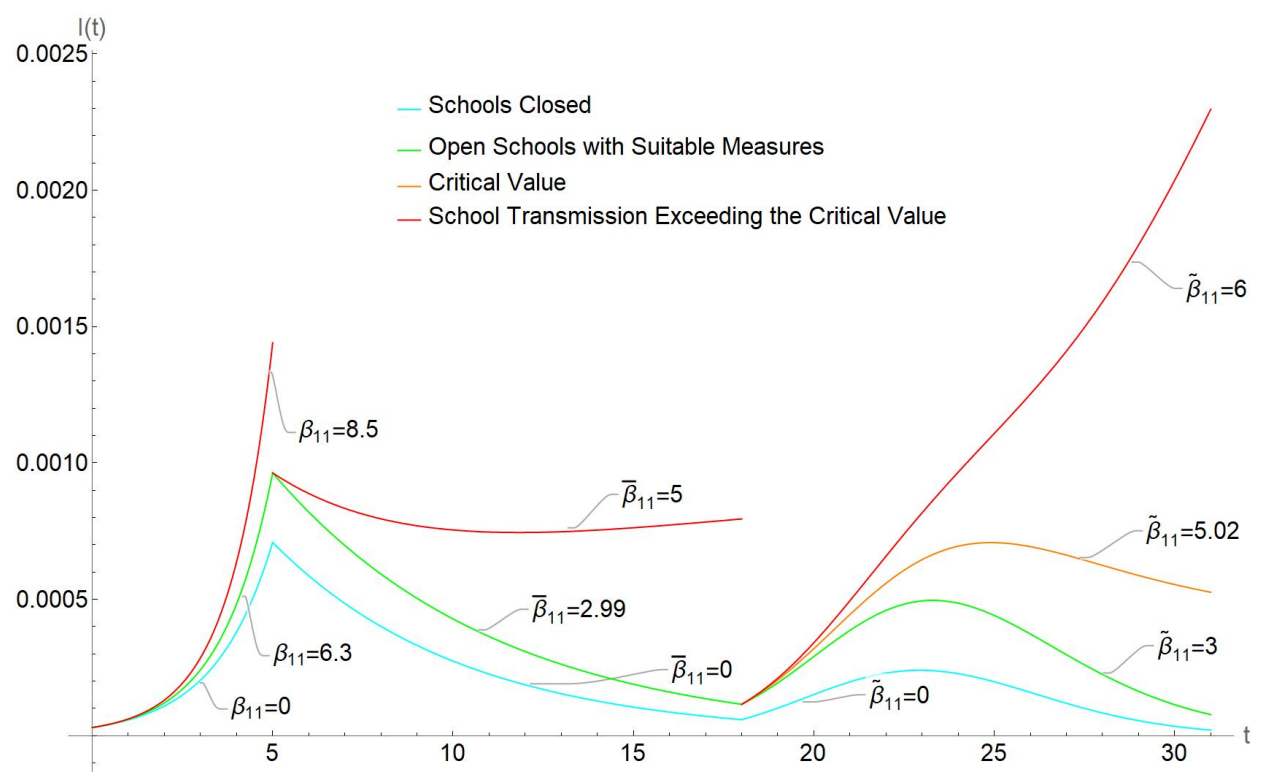

Figure 1: Daily active cases $I_{1}(t)+I_{2}(t)$ for various scenarios: outbreak or new strain upsurge, lockdown, and vaccination. In each case, there is critical value for the in-school transmission rate $\beta_{11}$. Cyan curve is with closed schools, green for safe opening, orange for critical values, red for values above criticality.

An analogous behavior takes place in more elaborate and realistic models, involving presymptomatic, asymptomatic, testing, isolation etc. Critical values appear for the in-school transmission rate, below which the effect of school opening on the epidemic trajectory is extremely contained. We provide simulations in Section A.15 (see Figure 11), and evidence from case studies here below. 


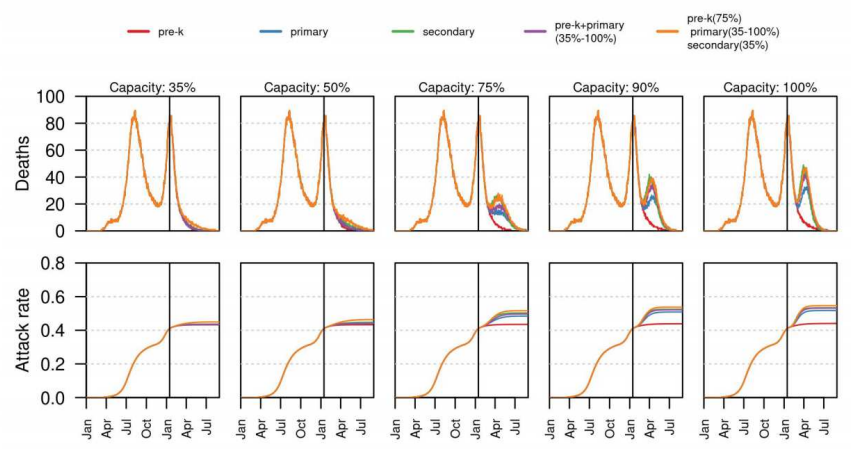

Figure 2: The impact of school reopening strategies in time as simulated in [33] from data from Bogotà, Colombia, for various values of the capacity, i.e. the percentage of students allowed back at school. Each column shows a different capacity level. Top panel shows the median daily incidence of deaths for each reopening strategy based on grades. Bottom panel shows the estimated attack rate for each of the reopening scenarios. Vertical black line shows the timing of school reopening (January 25, 2021). All scenarios were simulated up to August 31, 2021.

\subsection{Evidence of phase transition appears in several data driven case studies}

The effect of a phase transition seems to appear in all data driven studies (see Section A.16). In most studies the effect can be seen as the research reaches a definite conclusion: in some cases, the data or the model after calibration correspond to a subcritical regime, so that the study ends up asserting the almost irrelevance of school opening on the pattern of the epidemic for all the analyzed cases; in other cases, the study determines a supercritical setting, and then comes to the opposite conclusion.

Some works include one or more parameters that can be modulated to envision the effect of school reopening. In these cases, one can see the effect of a sharp transition from a subcritical, acceptable reopening, to an excessively impactful one. In [33], Figures 4 and 5, for instance, one can see that up to $50 \%$ capacity the effect of opening schools is almost negligible, while it becomes substantial above $75 \%$; this is a likely indication of a critical point between these values. For convenience, Figure 4 in [33] is reproduced here in Figure 2.

A very detailed study of school opening in The Netherlands is conducted in [9], and their conclusions are a clear description of the phase transition. Using a data driven, elaborate model, [9] claims that their "analyses suggest that the impact of measures reducing school-based contacts depends on the remaining opportunities to reduce non-school-based contacts. If opportunities to reduce the effective reproduction number $\left(R_{e}\right)$ with non-school-based measures are exhausted or undesired and $R_{e}$ is still close to 1 , the additional benefit of 
school-based measures may be considerable, particularly among older school children." The first scenario of [9] corresponds to a subcritical in-school transmission rate, so that the effect of closing schools would be very moderate. The second scenario seems to correspond to an in-school transmission rate around the critical value, so that both containment, in- or out-of-school, are effective.

[31] uses a detailed compartmental model and data from the second semester 2020 in Toronto, an outbreak context, to estimate the likely impact of school opening; with parameters estimated and collected from literature, the paper finds that opening school has little effect on the overall course of the pandemic: in all scenarios presented in Figures 2 and 4 the difference between school opening and closure is extremely contained. The findings of the research is then consistent with our phase transition scenario. In particular, [31] finds that "school reopening was not the key driver in virus resurgence, but rather it was community spread that determined the outbreak trajectory"; in other words, the parameters of the models, although not explicitly given in the paper, are such that the external transmission is preponderant. As an additional finding, it is observed in [31] that, according to their model, "brief school closures did reduce infections when transmission risk within the home was low": in this case, a reduced transmission rate outside makes the one in school likely supercritical.

\section{Confounding effects on retrospective studies and forecasting}

The presence of a threshold raises the specter of possible close to chaotic behaviors, a phenomenon affecting epidemic forecasts as outlined in [4]. Even with close-by initial data, if one of two systems crosses a critical threshold, then the future evolution might become extremely different from that of the other. For this reason, predictions on the future effects of in-school activities are going to be highly unreliable.

To make things worse, even retrospective studies trying to evaluate the role of school openings or closures on the evolution of the pandemic run the risk of being completely untrustworthy. Covid-19 data are affected by enormous errors, due to the presence of asymptomatic, lack and partial reliability of testing, difficulty in assessing close contacts etc. It follows that estimation of parameters for both statistical and model based studies are affected by large errors. In the presence of a threshold, even small errors can lead to incorrect attribution of the situation under observation to one phase, or to conflicting attributions to two opposite phases by different studies. In such scenario, a retrospective study could misclassify the effect of school opening or closure; and different studies even based on almost the same data might end up reaching opposite conclusions.

In the noisy, synthetic data in Figure 3, the number of daily infected in a population have been generated with the same parameters, except that

$$
\begin{aligned}
\text { first scenario: } & \beta_{11}=10, \beta_{2,2}=2, I_{2}(0)=3 \times 10^{-5} \\
\text { second scenario: } & \beta_{11}=6, \beta_{2,2}=2.57, I_{2}(0)=5.5 \times 10^{-6} .
\end{aligned}
$$




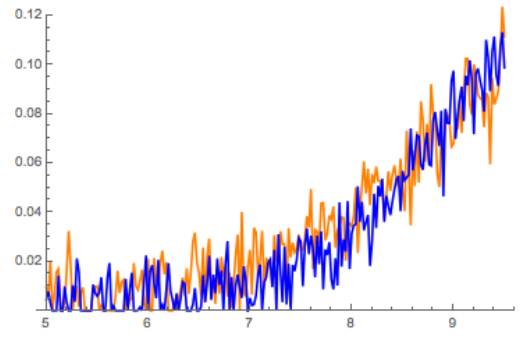

Figure 3: Synthetic data of daily active cases in the two different scenarios, one with subcritical and one with supercritical in-school transmission rates, and slightly different initial number of cases. Gaussian noise has been added to make the example more realistic.

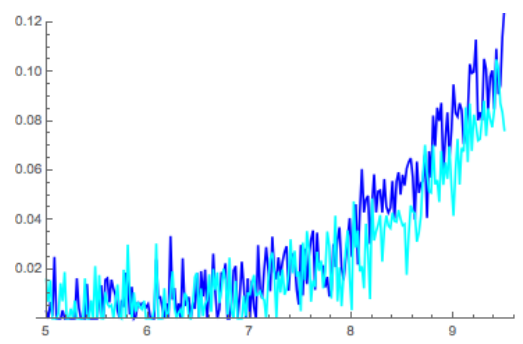

Figure 4: Reduction in daily cases due to school closure in the first scenario

In school transmission rates are supercritical in the first case, and subcritical in the second. But the different number of initial cases, a value that is subject to errors of various order of magnitudes and is quite arbitrarily assigned in the various studies, makes the two trajectory basically indistinguishable.

In a retrospective study one is forced to assign an initial value to the number of infected, and then estimate other parameters from the observations. Both scenarios are then plausible, depending on the chosen initial values. As Figure 4 confirms, the research would conclude in the first scenario, that closing schools would have been basically useless. In the second scenario, however, the opposite conclusion would be drawn, as illustrated by Figure 5 . 


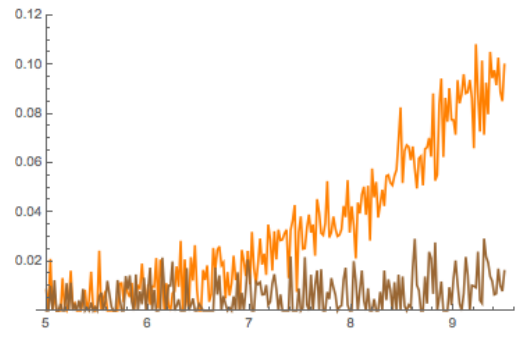

Figure 5: Reduction in daily cases due to school closure in the second scenario

\section{Conclusions}

Our results indicate that from the point of view of the effect of school opening on the overall course of the pandemic, there are no fundamental reasons to keep schools closed.

However, school opening comes at a price: the transmission rate in schools must be kept below a certain threshold that depends on the situation, and might not be easily determinable nor achievable.

The presence of a threshold is the likely cause of the opposing views that many studies have presented, between asserting almost irrelevance of school opening, and others pointing to its significant effects. Minimal changes in the overall conditions, or in values of the estimated parameters may determine one phase of the other; this may result in different attributions of responsibility to school opening, and creates the possibility of an arbitrary identification of the phase due to parameter estimation in the presence of very noisy data.

In particular, in the presence of a vaccination being carried out largely for out-of-school individuals only, there is a threshold below which schools can still be opened; it corresponds, however, to an internal reproduction number that would eradicate the virus if schools were completely isolated. As this is highly demanding, such a containment seems to be sustainable for brief periods only, after which vaccination for children becomes the only viable possibility to return to normality.

\section{$5 \quad$ Limitations and related works}

Although the presence of a phase transition in the effect of the school transmission rate in the overall course of the epidemic seems to have been unnoticed so far in the literature, there are many works related to ours.

In the direction opposite to the one taken here, namely focusing on the sustainability of opening from the point of containing the number of cases of a single school, one can see [11].

Compartmental models with two subpopulations are discussed in many works in general terms, see for instance a review in [20]; and then applied to the school 
opening issue in data driven analyses in $[26,31-33]$ : we discuss the relation of some these results with our work in Section A.16.

Finally, other papers $[24,26,27]$ make a purely statistical evaluation of the effect of school opening (see Section A.16).

Our work has several limitations. Our results are based on abstract, simplified models, and, although they seem to be stable when more detailed features are included, we cannot ensure that they always take place in more complex models.

Even when a critical value can be estimated, insuring that the transmission rates are below their relative thresholds is clearly a matter of distancing, testing, and other measures (see [https://science.sciencemag.org/content/369/6508/1146] for a qualitative list]). We do not elaborate here on how to develop a set of possible interventions, and on how to measure their success on containing the transmission rates in schools.

\section{References}

[1] Byrne, A. et al. Inferred duration of infectious period of SARS-CoV-2: rapid scoping review and analysis of available evidence for asymptomatic and symptomatic COVID-19 cases. BMJ Open 10, (2020).

[2] Bertozzi A. et al. The challenges of modeling and forecasting the spread of COVID-19, PNAS July 21, 2020117 (29) 16732-16738;

[3] Brauner et al. Inferring the effectiveness of government interventions against COVID-19, Science, 19 Feb 2021: Vol. 371, Issue 6531, DOI: 10.1126/science.abd9338

[4] Castro M., Ares S., Cuesta J. A., Manrubia S., The turning point and end of an expanding epidemic cannot be precisely forecast. Proceedings of the National Academy of Sciences Oct 2020, 117 (42) 26190-26196.

[5] Gandini et al. A cross-sectional and prospective cohort study of the role of schools in the SARS-CoV-2 second wave in Italy, The Lancet Regional Health - Europe, VOLUME 5, 100092, (2021)

[6] Gold JA, Gettings JR, Kimball A, et al. Clusters of SARS-CoV-2 Infection Among Elementary School Educators and Students in One School District - Georgia, December 2020-January 2021, MMWR Morb Mortal Wkly Rep 2021;70:289-292

[7] Roda W., Varughese M.B., Han D.,and Lia M., Why is it difficult to accurately predict the COVID-19 epidemic? Infect Dis Model. 2020; 5: 271-281. Published online 2020 Mar 25. doi: 10.1016/j.idm.2020.03.001

[8] Erdélyi A., Magnus W., Oberhettinger F. and Tricomi F., Higher Transcendental Functions Volume I, McGraw-Hill Book Company; 1st Edition (November 1, 1953.

[9] Rozhnova G. et al. Model-based evaluation of school- and non-school-related measures to control the COVID-19 pandemic, Nature Communications volume 12, Article number: 1614 (2021) 
[10] Prem K. et al. Projecting social contact matrices in 152 countries using contact surveys and demographic data. PLOS Computational Biology 13, (2017).

[11] Gandolfi, A. Planning of school teaching during Covid-19. Physica D: Nonlinear Phenomena 415, (2021).

[12] De la Sen, M., Ibeas, A. On an SE(Is)(Ih)AR epidemic model with combined vaccination and antiviral controls for COVID-19 pandemic. Adv Differ Equ 2021, 92 (2021). https://doi.org/10.1186/s13662-021-03248-5

[13] Lee, B. et al. Modeling the impact of school reopening on SARS-CoV-2 transmission using contact structure data from Shanghai. BMC Public Health 20, (2020).

[14] Viner, R. M. et al. School closure and management practices during coronavirus outbreaks including COVID-19: a rapid systematic review. The Lancet Child 85 Adolescent Health 4, 397-404 (2020).

[15] Walsh, S. et al. Do school closures reduce community transmission of COVID-19? A systematic review of observational studies. Preprint at https://www.medrxiv . org/content/10.1101/2021.01.02.21249146v1.full-text (2021).

[16] Cao, W. et al. The psychological impact of the COVID-19 epidemic on college students in China. Psychiatry Research 287, (2020).

[17] Libotte, G. B. et al. Determination of an optimal control strategy for vaccine administration in COVID-19 pandemic treatment. Computer Methods and Programs in Biomedicine 196, (2020).

[18] Singh, S. et al. Impact of COVID-19 and lockdown on mental health of children and adolescents: A narrative review with recommendations. Psychiatry Research 293 (2020).

[19] Panovska-Griffiths, J. et al. Determining the optimal strategy for reopening schools, the impact of test and trace interventions, and the risk of occurrence of a second COVID-19 epidemic wave in the UK: a modelling study. The Lancet Child 8 Adolescent Health 4, 817-827 (2020).

[20] Soyoung, K. et al. School opening delay effect on transmission dynamics of Coronavirus disease 2019 in Korea: Based on mathematical modeling and simulation study. Journal of Korean Medical Science 35, (2020).

[21] What we know about COVID-19 transmission in schools: the latest on the COVID-19 global situation \& the spread of COVID-19 in schools. World Health Organization Report, https://www.who.int/docs/default-source/ coronaviruse/risk-comms-updates/update39-covid-and-schools.pdf? sfvrsn=320db233_2 (October 2020).

[22] Brauer, F. Mathematical Epidemiology: Past, present, and future. Infectious Disease Modelling 2, 113-127 (2017).

[23] Keeling, M. J. et al. Modelling infectious diseases in humans and animals. Princeton University Press Chapter 3, (2008).

[24] Iwata, K. et al. Was school closure effective in mitigating coronavirus disease 2019 (COVID-19)? Time series analysis using Bayesian inference. International Journal of Infectious Diseases 99, 57-61 (2020).

[25] Matzinger, P. et al. Strong impact of closing schools, closing bars and wearing masks during the COVID-19 pandemic: results from a simple and revealing analysis. Preprint at https://www.medrxiv.org/content/10.1101/2020.09.26. 20202457v1.full-text (2020). 
[26] Ismail, S. A. et al. SARS-CoV-2 infection and transmission in educational settings: a prospective, cross-sectional analysis of infection clusters and outbreaks in England. The Lancet Infectious Diseases 21, 344-353 (2021).

[27] Larosa, E. et al. Secondary transmission of COVID-19 in preschool and school settings after their reopening in northern Italy: a population-based study. Preprint at https://www.medrxiv.org/content/10.1101/2020.11.17. 20229583v1.full-text (2020).

[28] Flaxman, S. et al. Estimating the effects of non-pharmaceutical interventions on COVID-19 in Europe. Nature 584 257-261 (2020).

[29] Singanayagam, A. et al. Duration of infectiousness and correlation with RT-PCR cycle threshold values in cases of COVID-19, England, January to May 2020. Euro Surveill 25, (2020).

[30] Blyuss, K. B. et al. Effects of Latency and Age Structure on the Dynamics and Containment of COVID-19. Journal of Theoretical Biology 513 (2021).

[31] Yuan, P. et al. School and community reopening during the COVID-19 pandemic: a mathematical modeling study. Preprint at https://www.medrxiv.org/ content/10.1101/2021.01.13.21249753v1.full (2021).

[32] Di Domenico, L. et al. Modelling safe protocols for reopening schools during the COVID-19 pandemic in France. Nature Communications 12 (2021).

[33] España, G. et al. The impact of school reopening on COVID-19 dynamics in Bogotá, Colombia. Preprint at https://osf.io/ebjx9/ (2021).

[34] Keskinocak, P., Asplund, J., Serban, N. \& Oruc Aglar, B. E. Evaluating scenarios for school reopening under COVID19. Preprint at medRxiv https://doi.org/10.1101/2020.07.22.20160036 (2020).

[35] Voinsky, I. et al. Effects of age and sex on recovery from COVID-19: Analysis of 5769 Israeli patients. The Journal of Infection 81, (2020).

[36] Oran, D.P., Topol, E.J. Prevalence of Asymptomatic SARS-CoV-2 Infection. Annals of Internal Medicine 173, (2020).

[37] Davies, N.G. et al. Age-dependent effects in the transmission and control of COVID-19 epidemics. Nature Medicine 26, (2020).

[38] Dong, Y. et al Epidemiology of COVID-19 Among Children in China. Pediatrics 145, (2020).

\section{A Methods}

\section{A.1 Compartmental models}

In order to evaluate the effect of school opening onto the course of the epidemic we use compartmental models, as they proved capable of predicting the courses of outbreaks in many instances [22]. We start with the simplest SIR model with unit total population, and two subclasses of sizes $n_{1}$ and $n_{2}$. More features are added later on. We make a sequence of theoretical claims concerning the effect of the contact rate in the subclass representing schools. Most of the claims are verified in suitable linear approximations of the SIR model; these give very close approximations of non linear versions as in the entire course of the current COVID-19 pandemic the proportion of active cases $I=I_{1}+I_{2}$ is kept relatively low by either containment measures, lockdowns, or vaccinations. Each result is then complemented with numerical simulations. 


\section{A.2 SIR model with two subclasses}

We first consider the simplest model of interest, represented in terms of a coupled SIR system

$$
\left\{\begin{array}{l}
S_{1}^{\prime}=-\beta_{11} S_{1} I_{1}-\beta_{12} S_{1} I_{2} \\
I_{1}^{\prime}=\beta_{11} S_{1} I_{1}+\beta_{12} S_{1} I_{2}-I_{1} \\
R_{1}^{\prime}=I_{1} \\
S_{2}^{\prime}=-\beta_{21} S_{2} I_{1}-\beta_{22} S_{2} I_{2} \\
I_{2}^{\prime}=\beta_{21} S_{2} I_{1}+\beta_{22} S_{2} I_{2}-I_{2} \\
R_{2}^{\prime}=I_{2}
\end{array}\right.
$$

Notice that the recovery rate is the same in the two subpopulations as for COVID-19 they seem to depend on the severity of the infection but not directly on age [1], [29], [30], and time is rescaled so that it is equal to 1 . This makes time unit of about 10-14 days [35]. In addition, $\beta_{12}, \beta_{21}$ are generally smaller than $\beta_{11}, \beta_{22}$ [10], [20]. We intend to compare the attack rates $\Delta S\left(\beta_{11}\right)=S_{1}\left(t_{a}\right)-S_{1}\left(t_{b}\right)+S_{2}\left(t_{a}\right)-S_{2}\left(t_{b}\right)$ between two suitable times $t_{a}<t_{b}$, as function of the in-school transmission rate $\beta_{11}$; here $\beta_{11}=0$ corresponds to schools being closed, and $\beta_{11}>0$ corresponds to schools being open with varying degrees of physical distancing and other containment measures in place.

\section{A.3 Linear approximation during the initial phase of an outbreak or new strain upsurge}

A suitable linear approximation for the initial period of the first outbreak, or of any of the possible infection waves taking place after a successful lockdown, is the following

$$
\left\{\begin{array}{l}
S_{1}^{\prime}=-\beta_{11} S_{1}(0) I_{1}-\beta_{12} S_{1}(0) I_{2} \\
I_{1}^{\prime}=\left(\beta_{11} S_{1}(0)-1\right) I_{1}+\beta_{12} S_{1}(0) I_{2} \\
S_{2}^{\prime}=-\beta_{21} S_{2}(0) I_{1}-\beta_{22} S_{2}(0) I_{2} \\
I_{2}^{\prime}=\beta_{21} S_{2}(0) I_{1}+\left(\beta_{22} S_{2}(0)-1\right) I_{2}
\end{array}\right.
$$

from which we extract the second and the fourth equations for $I_{1}, I_{2}$. In vector form we have

$$
\vec{I}^{\prime}=(A-\mathrm{Id}) \vec{I}
$$

where Id is the $2 \times 2$ identity matrix and

$$
A=\left[\begin{array}{ll}
a_{11} & a_{12} \\
a_{21} & a_{22}
\end{array}\right]:=\left[\begin{array}{ll}
\beta_{11} S_{1}(0) & \beta_{12} S_{1}(0) \\
\beta_{21} S_{2}(0) & \beta_{22} S_{2}(0)
\end{array}\right]
$$

is the reproduction matrix [23].

Lemma A.1. The solution of (11) is

$$
\vec{I}=e^{-t} e^{A t} \overrightarrow{I_{0}}=e^{-t}\left(e^{\lambda_{\max } t} \vec{W}+e^{\lambda_{\min } t} \vec{V}\right)
$$

where $\lambda_{\text {max }}, \lambda_{\text {min }}$ are the positive eigenvalues of the matrix $A$, and $\vec{W}>0$.

The proof is in Appendix B.

The largest eigenvalue of $A$ is the overall reproduction number $R_{0}$, [23], and the early evolution of the epidemics depends on the size of $\lambda_{\max }$, and on the spectral gap $\lambda_{\max }-\lambda_{\min }$. This is the so-called slaved phase, in which the active cases of both populations are both lead by approximately the same exponential growth [23]. 


\section{A.4 Dependence of the largest eigenvalue of $2 \times 2$ matrices from the first entry}

To get a first indication of a sudden change in the effect of the in-school transmission rate $\beta_{11}$, we study the behavior of the largest eigenvalue of a quite general $2 \times 2$ matrix as function of its first entry. Let

$$
\lambda^{\prime}\left(a_{11}\right):=\frac{d \lambda_{\max }}{d a_{11}}
$$

then the following estimate holds:

Theorem A.2. Let $A=\left(a_{i j}\right)$ be a $2 \times 2$ matrix with positive entries and let $\lambda_{\max }\left(a_{11}\right)>$ $\lambda_{\min }\left(a_{11}\right)$ be its eigenvalues. We have that for any $\alpha \in\left(0, \frac{a_{22}}{\sqrt{a_{21} a_{12}}}\right)$

$$
\begin{aligned}
& \frac{\lambda_{\max }^{\prime}\left(a_{22}+\alpha \sqrt{a_{12} a_{21}}\right)-\lambda_{\max }^{\prime}\left(a_{22}-\alpha \sqrt{a_{12} a_{21}}\right)}{\lambda_{\max }^{\prime}\left(a_{22}-\alpha \sqrt{a_{12} a_{21}}\right)-\lambda_{\max }^{\prime}(0)}=: \frac{\Delta_{1}}{\Delta_{0}} \\
& =\frac{\Delta_{1}}{\Delta_{2}}=\frac{\lambda_{\max }^{\prime}\left(a_{22}+\alpha \sqrt{a_{12} a_{21}}\right)-\lambda_{\max }^{\prime}\left(a_{22}-\alpha \sqrt{a_{12} a_{21}}\right)}{\lambda_{\max }^{\prime}\left(2 a_{22}\right)-\lambda_{\max }^{\prime}\left(a_{22}+\alpha \sqrt{a_{12} a_{21}}\right)} \geq \frac{2 \alpha}{\sqrt{4+\alpha^{2}}-\alpha}
\end{aligned}
$$

The proof is in Appendix C. Figure 6 shows an example of the change in derivatives.

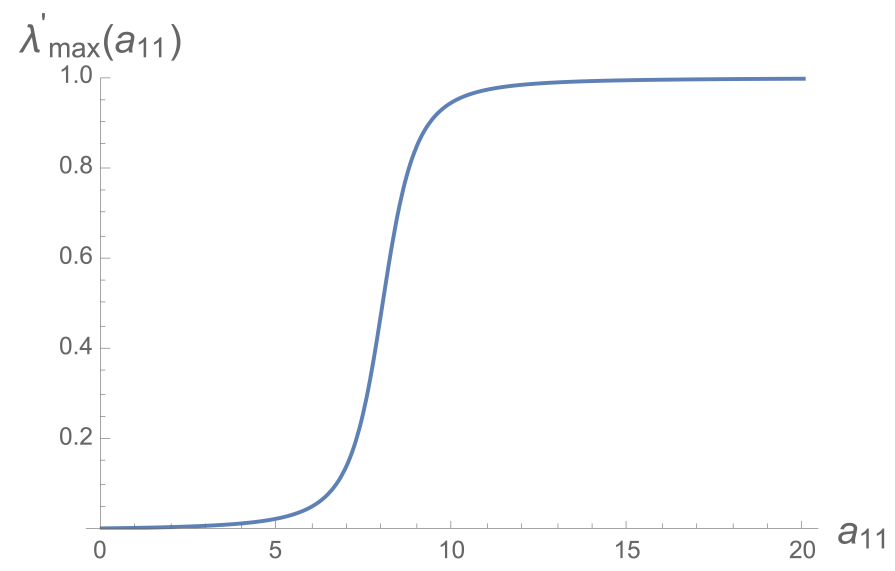

Figure 6: Derivative of the largest eigenvalue of $A=\left(a_{i j}\right)$ with respect to $a_{11}$, with $a_{12}=a_{21}=0.5$ and $a_{22}=8$.

As a consequence, if, for instance, $\alpha=2$ as we will assume from now on, then

$$
\Delta_{1} \geq 4.8 \Delta_{0}=4.8 \Delta_{2}
$$

Simple calculations in Appendix E show then that if $0<a_{11} \leq a_{22}-\alpha \sqrt{a_{12} a_{21}}$, which is realistic since $a_{22}^{2}>>a_{12} a_{21}$,

$$
\lambda_{\max }\left(2 a_{22}\right)-\lambda_{\max }(0) \geq 4.8\left(\lambda_{\max }\left(a_{11}\right)-\lambda_{\max }(0)\right) .
$$

The change in the largest eigenvalue is illustrated in Figure 7. 


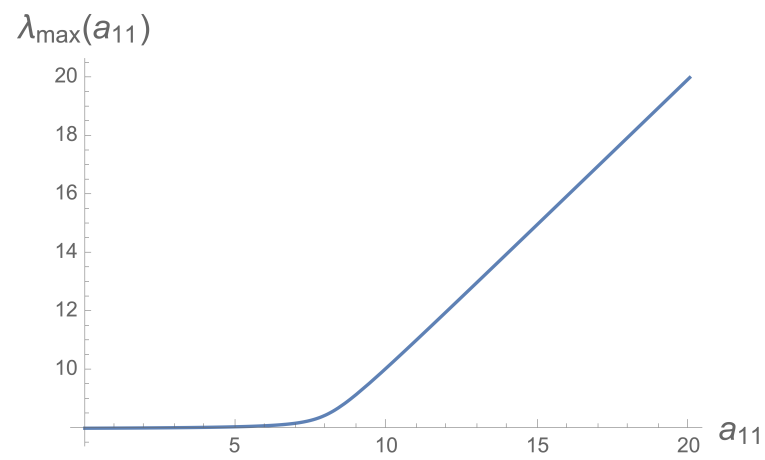

Figure 7: The largest eigenvalue of $A=\left(a_{i j}\right)$ as function of $a_{11}$, with $a_{12}=$ $a_{21}=0.5$ and $a_{22}=8$.

\section{A.5 A phase transition for school opening during an out- break}

We now want to see a similar behavior in the active cases $I_{1}+I_{2}$ in the coupled SIR model (9). Let

$$
\begin{aligned}
& \lambda_{\max }(0)=\lambda_{0}, \quad \lambda_{\min }(0) \quad=\widetilde{\lambda_{0}} \\
& \lambda_{\max }\left(a_{22}-\alpha \sqrt{a_{12} a_{21}}\right)=\lambda_{1}, \quad \lambda_{\min }\left(a_{22}-\alpha \sqrt{a_{12} a_{21}}\right) \quad=\widetilde{\lambda_{1}} \\
& \lambda_{\max }\left(a_{22}+\alpha \sqrt{a_{12} a_{21}}\right)=\lambda_{2}, \quad \lambda_{\min }\left(a_{22}+\alpha \sqrt{a_{12} a_{21}}\right)=\widetilde{\lambda_{2}} .
\end{aligned}
$$

and denote by $\vec{W}^{i}=\left(W_{1}^{i}, W_{2}^{i}\right)$ and $\vec{V}^{i}=\left(V_{1}^{i}, V_{2}^{i}\right)$, for $i=0,1,2$, the vectors $\vec{W}$ and $\vec{V}$ in (13) corresponding to $\lambda_{0}, \lambda_{1}, \lambda_{2}$, and $\widetilde{\lambda_{0}}, \widetilde{\lambda_{1}}, \widetilde{\lambda_{2}}$, respectively. Note that, without loss of generality, we can assume that $V_{1}^{i}>0$, for $i=0,1,2$.

Let $\vec{I}^{\lambda_{j}}(t), j=0,1,2$ denote the infected at time $t$ corresponding to eigenvalues $\lambda_{j}, j=0,1,2$ respectively.

Corollary A.3. For all $k>0$, there exists $T_{k}>0$ such that for all $t \geq T_{k}$

$$
\left|\vec{I}^{\lambda_{2}}(t)-\vec{I}^{\lambda_{0}}(t)\right| \geq k\left|\vec{I}^{\lambda_{1}}(t)-\vec{I}^{\lambda_{0}}(t)\right| .
$$

The proof is in Appendix D. This identifies $a_{22}$ as the critical point for the effect of the coefficient $a_{11}$ on the largest eigenvalue of $A$; by the relations in (12), the critical point for the effect of $\beta_{11}$ on the overall pandemic is then $\beta_{11}^{*}=\beta_{22} S_{2}(0) / S_{1}(0)$.

\section{A.6 Containment of the effect of school opening during an outbreak}

For $i=1,2$ and some $\bar{t}>0$, let

$$
\mathcal{I}_{i}=\int_{0}^{\bar{t}} I_{i}(\tau) d \tau
$$


Integrating the first and third equations of (10), we get

$$
\begin{aligned}
\Delta S_{i} & =S_{i}(0)-S_{i}(\bar{t})=-\int_{0}^{\bar{t}} S_{i}^{\prime}(\tau) d \tau \\
& =\int_{0}^{\bar{t}}\left(I_{i}^{\prime}(\tau)+I_{i}(\tau)\right) d \tau \\
& =I_{i}(\bar{t})-I_{i}(0)+\mathcal{I}_{i} .
\end{aligned}
$$

On the other hand, integrating the second and fourth equations of $(10)$ in $[0, \bar{t}]$, we get

$$
\left\{\begin{array}{l}
I_{1}(\bar{t})-I_{1}(0)=\left(\beta_{11} S_{1}(0)-1\right) \mathcal{I}_{1}+\beta_{12} S_{1}(0) \mathcal{I}_{2} \\
I_{2}(\bar{t})-I_{2}(0)=\beta_{21} S_{2}(0) \mathcal{I}_{1}+\left(\beta_{22} S_{2}(0)-1\right) \mathcal{I}_{2}
\end{array}\right.
$$

whose solution is

$$
\left\{\begin{array}{l}
\mathcal{I}_{1}=-\frac{I_{1}(0)-I_{1}(\bar{t})+\beta_{12}\left(I_{2}(0)-I_{2}(\bar{t})\right) S_{1}(0)-\beta_{22}\left(I_{1}(0)-I_{1}(\bar{t})\right) S_{2}(0)}{-1+\beta_{11} S_{1}(0)\left(1-\beta_{22} S_{2}(0)\right)+\beta_{22} S_{2}(0)+\beta_{12} \beta_{21} S_{1}(0) S_{2}(0)} \\
\mathcal{I}_{2}=-\frac{I_{2}(0)-I_{2}(\bar{t})-\beta_{11}\left(I_{2}(0)-I_{2}(\bar{t})\right) S_{1}(0)+\beta_{21}\left(I_{1}(0)-I_{1}(\bar{t})\right) S_{2}(0)}{-1+\beta_{11} S_{1}(0)\left(1-\beta_{22} S_{2}(0)\right)+\beta_{22} S_{2}(0)+\beta_{12} \beta_{21} S_{1}(0) S_{2}(0)}
\end{array}\right.
$$

The attack rate is then

$$
\begin{aligned}
A\left(\beta_{11}\right):= & \frac{1}{S_{1}(0)+S_{2}(0)}\left(\Delta S_{1}+\Delta S_{2}\right) \\
= & \frac{1}{S_{1}(0)+S_{2}(0)}\left(I_{1}(\bar{t})-I_{1}(0)+\mathcal{I}_{1}+I_{2}(\bar{t})-I_{2}(0)+\mathcal{I}_{2}\right) \\
= & \frac{1}{S_{1}(0)+S_{2}(0)}\left\{\left(\beta_{11} \beta_{22} S_{1}(0) S_{2}(0)\right.\right. \\
& \left.\quad-\beta_{12} \beta_{21} S_{1}(0) S_{2}(0)\right)\left[I_{1}(\bar{t})-I_{1}(0)+I_{2}(\bar{t})-I_{2}(0)\right] \\
& +\left(\beta_{21} S_{2}(0)+\beta_{11} S_{1}(0)\right)\left[I_{1}(0)-I_{1}(\bar{t})\right] \\
& \left.+\left(\beta_{12} S_{1}(0)+\beta_{22} S_{2}(0)\right)\left[I_{2}(0)-I_{2}(\bar{t})\right]\right\} \\
& \quad \times\left\{1-\beta_{11} S_{1}(0)\left(1-\beta_{22} S_{2}(0)\right)-\beta_{22} S_{2}(0)-\beta_{12} \beta_{21} S_{1}(0) S_{2}(0)\right\}^{-1}
\end{aligned}
$$

Notice that, to the contrary of what happens in the next section for the lockdown case, the value of $\beta_{11}$ for which the denominator is zero does not correspond to a singularity: this is to be expected as it differs from $\beta_{11}^{*}$, and we confirmed it numerically.

Taking $\vec{I}$ as in (13), we get an explicit expression for $\Delta S\left(\beta_{11}\right)$. For a fixed $\epsilon$, representing the allowed fractional increment in the number of cases when the school is open, the allowed bound for $\beta_{11}$ is given by

$$
A\left(\beta_{11}\right) / A(0) \leq 1+\epsilon .
$$

With the parameters used in Section 2.1, this gives the mentioned value $\beta_{11} \leq 6.344$.

\section{A.7 SIR for lockdown and its linear approximation}

System (9) is suitable to model lockdown as well, provided that the reproduction rate, which is the largest eigenvalue of (12), satisfies $R_{0}<1$, and that initial conditions taken at time $\bar{t}$ have a more substantial number of cases and recovered. A linear approximation of the system is possible as the overall number of active cases is never 
allowed to grow beyond relatively small fractions of the population, never more than $0.1 \%$ in most countries.

With these conditions, a linear approximation is

$$
\left\{\begin{array}{l}
I_{1}^{\prime}=\bar{\beta}_{11} S_{1}(\bar{t}) I_{1}+\bar{\beta}_{12} S_{1}(\bar{t}) I_{2}-I_{1} \\
I_{2}^{\prime}=\bar{\beta}_{21} S_{2}(\bar{t}) I_{1}+\bar{\beta}_{22} S_{2}(\bar{t}) I_{2}-I_{2} \\
I_{1}(\bar{t}), I_{2}(\bar{t})>0
\end{array}\right.
$$

with $S_{1}(\bar{t})+S_{2}(\bar{t})+I_{1}(\bar{t})+I_{2}(\bar{t})<1$.

Figure 8 illustrates via a simulation for $S_{1}(\bar{t})=0.15<0.2, S_{2}(\bar{t})=0.7<0.8, I_{1}(\bar{t})=$ $I_{2}(\bar{t})=10^{-4}, \bar{\beta}_{12}=\bar{\beta}_{21}=0.25, \bar{\beta}_{22}=1$, and $\bar{\beta}_{11}=0,3,5$, the closeness of the linear approximation. The total number of cases simulated from the differential system and the linear approximation are not distinguishable in the figure for all values of $\bar{\beta}_{11}$; the same holds for each subpopulation.

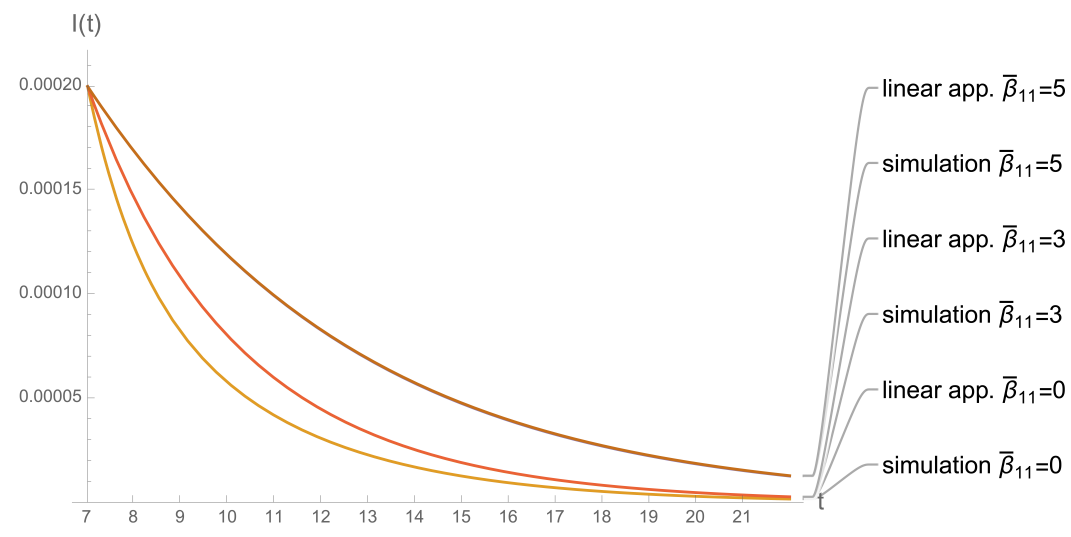

Figure 8: Effectiveness of the linear approximation of the SIR model for lockdown; the figure shows the total active cases numerically simulated with realistic parameters and varying $\bar{\beta}_{11}$ : in each test, simulations from the differential system and from its linear approximation are indistinguishable.

\section{A.8 Allowed level of school transmission for a successful lockdown}

Let us assume that a lockdown is applied from time $\bar{t}$ to $\tilde{t}$ that successfully eradicate the virus; hence with $I_{i}(\tilde{t}) \approx 0$ for $i=1,2$. From the mathematical point of view, we can take the eradication time to be $+\infty$ as the dynamical system reaches an equilibrium with no active cases and does not change afterwards. Hence we consider $\Delta S_{i}=$ $S_{i}(\infty)-S_{i}(\tilde{t}) \approx S_{i}(\bar{t})-S_{i}(\tilde{t})$. Formulas (16) -(19) apply, with 0 and $\bar{t}$ replaced by $\bar{t}$ and $\infty$, respectively; the quantities $\mathcal{I}_{i}, \beta_{i, j}$, for $i, j=1,2$, decorated by an overscore; and $I_{1}(\infty)=I_{2}(\infty)=0$.

The denominator of (19) with the above changes is singular for

$$
\bar{\beta}_{11}^{*}=\frac{1}{S_{1}(\bar{t})}-\frac{\bar{\beta}_{12} \bar{\beta}_{21} S_{2}(\bar{t})}{\left(1-\bar{\beta}_{22} S_{2}(\bar{t})\right)} .
$$


The numerator at $\bar{\beta}_{11}=\bar{\beta}_{11}^{*}$, on the other hand, is not identically zero; this is seen by substituting the value (22) for $\bar{\beta}_{11}$ in (19), with the adaptations listed above: after some algebra, carried out in Mathematica ${ }^{\mathrm{TM}}$, the numerator is seen to equal

$$
\frac{\left(1+\bar{\beta}_{21} S_{2}(\bar{t})\right)\left(\bar{\beta}_{12} I_{2}(\bar{t}) S_{1}(\bar{t})+I_{1}(\bar{t})\left(1-\bar{\beta}_{22} S_{2}(\bar{t})\right)\right)}{\left(1-\bar{\beta}_{22} S_{2}(\bar{t})\right)} \approx-1.6 \times 10^{-5} ;
$$

the numerical value is computed with the values indicated in Section 2.2, namely that

$$
\bar{\beta}_{12}=\bar{\beta}_{21}=0.25, \bar{\beta}_{22}=1, \epsilon=0.3
$$

and choosing as initial condition at $t=\bar{t}=5$ the total number of susceptible and infected obtained from the outbreak scenario, that is

$$
S_{1}(\bar{t}) \approx 0.1996, S_{2}(\bar{t}) \approx 0.7981, I_{1}(\bar{t}) \approx 1.601 \times 10^{-4}, I_{2}(\bar{t}) \approx 7.9555 \times 10^{-4} .
$$

This indicates that the value in (22) is where the linear approximation breaks down, indicating a transition of phases.

In addition, if we require that school opening does not affect more than a certain percentage the overall incidence proportion by asking that

$$
A\left(\bar{\beta}_{11}\right) \leq(1+\epsilon) A(0)
$$

for some $\epsilon>0$, then after some algebra, carried out in Mathematica ${ }^{\mathrm{TM}}$, we get that

$$
\begin{aligned}
\bar{\beta}_{11} \leq F(\epsilon) & =\left[\epsilon ( - 1 + \overline { \beta } _ { 2 2 } S _ { 2 } ( \overline { t } ) + \overline { \beta } _ { 1 2 } \overline { \beta } _ { 2 1 } S _ { 1 } ( \overline { t } ) S _ { 2 } ( \overline { t } ) ) \left(\bar{\beta}_{12} I_{2}(\bar{t}) S_{1}(\bar{t})\right.\right. \\
& +\bar{\beta}_{21} I_{1}(\bar{t}) S_{2}(\bar{t})+\bar{\beta}_{22} I_{2}(\bar{t}) S_{2}(\bar{t})+\bar{\beta}_{12} \bar{\beta}_{21} I_{1}(\bar{t}) S_{1}(\bar{t}) S_{2}(\bar{t}) \\
& \left.\left.+\bar{\beta}_{12} \bar{\beta}_{21} I_{2}(\bar{t}) S_{1}(\bar{t}) S_{2}(\bar{t})\right)\right] \times\left\{S _ { 1 } ( \overline { t } ) \left[-I_{1}(\bar{t})-\bar{\beta}_{12} I_{2}(\bar{t}) S_{1}(\bar{t})\right.\right. \\
& -\epsilon \bar{\beta}_{12} I_{2}(\bar{t}) S_{1}(\bar{t})-\bar{\beta}_{21} I_{1}(\bar{t}) S_{2}(\bar{t})+2 \bar{\beta}_{22} I_{1}(\bar{t}) S_{2}(\bar{t})-\epsilon \bar{\beta}_{21} I_{1}(\bar{t}) S_{2}(\bar{t}) \\
& -\epsilon \bar{\beta}_{22} I_{2}(\bar{t}) S_{2}(\bar{t})-\epsilon \bar{\beta}_{12} \bar{\beta}_{21} I_{1}(\bar{t}) S_{1}(\bar{t}) S_{2}(\bar{t})-\bar{\beta}_{12} \bar{\beta}_{21} I_{2}(\bar{t}) S_{1}(\bar{t}) S_{2}(\bar{t}) \\
& +\bar{\beta}_{12} \bar{\beta}_{22} I_{2}(\bar{t}) S_{1}(\bar{t}) S_{2}(\bar{t})-\epsilon \bar{\beta}_{12} \bar{\beta}_{21} I_{2}(\bar{t}) S_{1}(\bar{t}) S_{2}(\bar{t}) \\
& +\epsilon \bar{\beta}_{12} \bar{\beta}_{22} I_{2}(\bar{t}) S_{1}(\bar{t}) S_{2}(\bar{t})+\bar{\beta}_{21} \bar{\beta}_{22} I_{1}(\bar{t}) S_{2}^{2}(\bar{t})-\bar{\beta}_{22}^{2} I_{1}(\bar{t}) S_{2}^{2}(\bar{t}) \\
& +\epsilon \bar{\beta}_{21} \bar{\beta}_{22} I_{1}(\bar{t}) S_{2}^{2}(\bar{t})+\epsilon \bar{\beta}_{22}^{2} I_{2}(\bar{t}) S_{2}^{2}(\bar{t}) \\
& \left.\left.+\epsilon \bar{\beta}_{12} \bar{\beta}_{21} \bar{\beta}_{22} I_{1}(\bar{t}) S_{1}(\bar{t}) S_{2}^{2}(\bar{t})+\epsilon \bar{\beta}_{12} \bar{\beta}_{21} \bar{\beta}_{22} I_{2}(\bar{t}) S_{1}(\bar{t}) S_{2}^{2}(\bar{t})\right]\right\}^{-1}
\end{aligned}
$$

With the values as in $(24)$ and $(25)$ we get $F(0.3) \approx 2.9944$.

We compare this expression with the bound in (2), in some numerical examples.

\section{A.9 A complete outbreak-lockdown cycle}

A confirmation of the behavior of the effect of school opening on one outbreak-lockdown cycle is shown here via a direct simulation.

Continuing the numerical example of Section 2.2, suppose a lockdown is imposed starting from $\bar{t}=5$, and a $50 \%$ reduction is achieved in the transmission rates different from $\beta_{11}$; Figure $1, t \in[5,18]$, shows that as the outbreak is resolved after the lockdown, the cumulative number of cases is close to that at $\bar{\beta}_{11}=0$ when $\beta_{11}$ and $\bar{\beta}_{11}$ are close enough to the origin, and sharply deviates otherwise. 


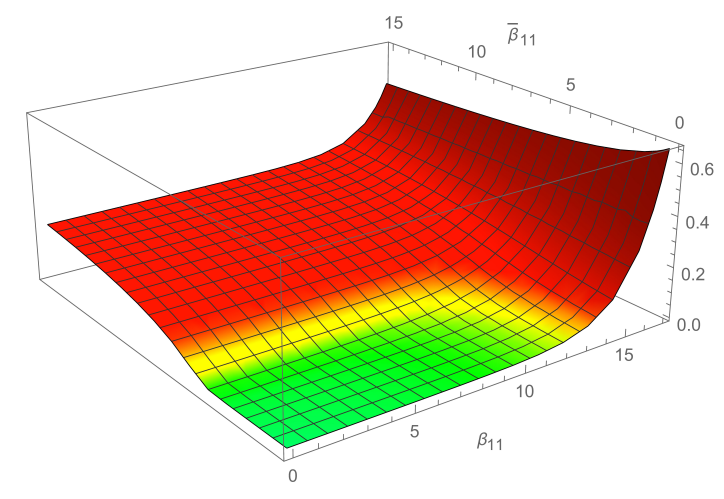

Figure 9: Total number of cases at resolution of outbreak for varying values of $\beta_{11}$ and $\bar{\beta}_{11}$.

\section{A.10 SIR with two subpopulations and vaccination}

Vaccination is included in the model assuming that the adult population is vaccinated at a constant rate $v$ :

$$
\left\{\begin{array}{l}
S_{1}^{\prime}=-\widetilde{\beta}_{11} S_{1} I_{1}-\widetilde{\beta}_{12} S_{1} I_{2} \\
I_{1}^{\prime}=\widetilde{\beta}_{11} S_{1} I_{1}+\widetilde{\beta}_{12} S_{1} I_{2}-I_{1} \\
R_{1}^{\prime}=I_{1} \\
S_{2}^{\prime}=-\widetilde{\beta}_{21} S_{2} I_{1}-\widetilde{\beta}_{22} S_{2} I_{2}-v S_{2} \\
I_{2}^{\prime}=\widetilde{\beta}_{21} S_{2} I_{1}+\widetilde{\beta}_{22} S_{2} I_{2}-I_{2} \\
R_{2}^{\prime}=I_{2}+v S_{2}
\end{array}\right.
$$

see, e.g. [17], [12]. Here, for seek of simplicity we assume that the time $\tilde{t}$ at which the vaccination program begins corresponds to $t=0$.

We are not aware of a way to explicitly express the solutions of this system.

\section{A.11 A linear approximation to SIR with vaccination}

In order to analyze (27) we develop a linearization. Notice that in the linear approximation for the initial phase of an SIR model, the terms $\widetilde{\beta}_{i 1} S_{i} I_{1}+\widetilde{\beta}_{i 2} S_{i} I_{2}$ are taken to be zero for both $i=1,2$. With the same assumption in the vaccination case, we get the equation $S_{2}^{\prime}=-v S_{2}$ : we therefore use the solution to this equation as linear approximation of $S_{2}(t)$. This leads to the following linearization

$$
\left\{\begin{array}{l}
S_{1}^{\prime}=-\widetilde{\beta}_{11} S_{1}(0) I_{1}-\widetilde{\beta}_{12} S_{1}(0) I_{2} \\
I_{1}^{\prime}=\widetilde{\beta}_{11} S_{1}(0) I_{1}+\widetilde{\beta}_{12} S_{1}(0) I_{2}-I_{1} \\
R_{1}^{\prime}=I_{1} \\
S_{2}^{\prime}=-\widetilde{\beta}_{21} S_{2}(0) e^{-v t} I_{1}-\widetilde{\beta}_{22} S_{2}(0) e^{-v t} I_{2}-v S_{2} \\
I_{2}^{\prime}=\widetilde{\beta}_{21} S_{2}(0) e^{-v t} I_{1}+\widetilde{\beta}_{22} S_{2}(0) e^{-v t} I_{2}-I_{2} \\
R_{2}^{\prime}=I_{2}+v S_{2} .
\end{array}\right.
$$


From (28) we extract

$$
\left\{\begin{array}{l}
I_{1}^{\prime}=\widetilde{\beta}_{11} S_{1}(0) I_{1}+\widetilde{\beta}_{12} S_{1}(0) I_{2}-I_{1} \\
I_{2}^{\prime}=\widetilde{\beta}_{21} S_{2}(0) e^{-v t} I_{1}+\widetilde{\beta}_{22} S_{2}(0) e^{-v t} I_{2}-I_{2} .
\end{array}\right.
$$

Figure 10 shows one instance of the effectiveness of the linear approximation with realistic parameters.

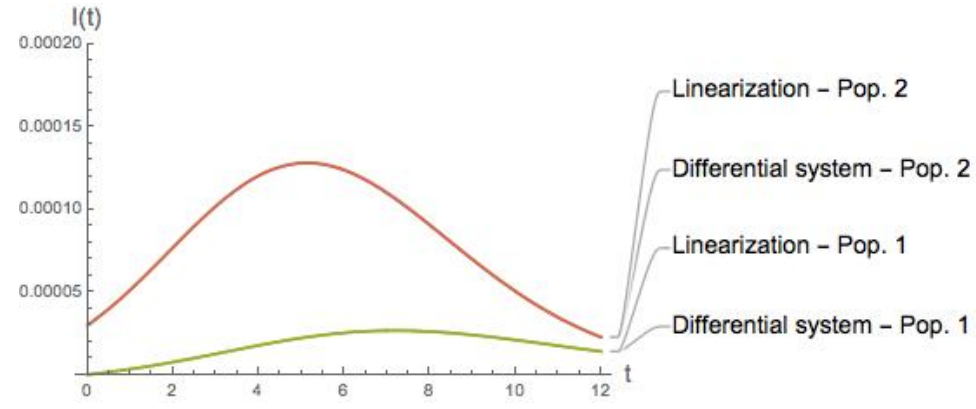

Figure 10: Effectiveness of the linear approximation of the SIR model with vaccination; the figure shows the total active cases numerically simulated with realistic parameters, $\widetilde{\beta}_{11}=3, \widetilde{\beta}_{22}=2$ : simulations from the differential system and from its linear approximation are indistinguishable.

\section{A.12 Evidence of a phase transition in $\widetilde{\beta}_{11}$ with critical point $1 / S_{1}(0)$ during vaccination}

We proceed by using the linear approximation to evaluate the attack rates as function of $\widetilde{\beta}_{11}$; in particular, we focus on the one for the external population 2. Our calculation is done recursively, as shown in Appendix F. The following theorem summarizes the calculation.

Theorem A.4. Assume that $I_{1}, I_{2}$ are integrable in $[0,+\infty)$ and let

$$
\begin{aligned}
\widetilde{\mathcal{I}}_{1} & =\widetilde{\mathcal{I}}_{1}\left(\widetilde{\beta}_{11}\right)=\int_{0}^{+\infty} I_{1}(t) d t, \quad \widetilde{\mathcal{I}}_{2}=\widetilde{\mathcal{I}}_{2}\left(\widetilde{\beta}_{11}\right)=\int_{0}^{+\infty} I_{2}(t) d t \\
p_{k+1} & =I_{2}(0)+\frac{\widetilde{\beta}_{21} S_{2}(0) I_{1}(0)}{(k+1) v-\widetilde{\beta}_{11} S_{1}(0)+1} \\
q_{k+1} & =\widetilde{\beta}_{22} S_{2}(0)+\frac{\left.\widetilde{\beta}_{12} \widetilde{\beta}_{21} S_{1}(0) S_{2}(0)\right)}{(k+1) v-\widetilde{\beta}_{11} S_{1}(0)+1}
\end{aligned}
$$


for $k=0,1, \ldots$ We have that

$$
\begin{aligned}
& \widetilde{\mathcal{I}}_{1}=\widetilde{\mathcal{I}}_{1}\left(\widetilde{\beta}_{11}\right)=\frac{\widetilde{\beta}_{12} S_{1}(0) \widetilde{\mathcal{I}}_{2}+I_{1}(0)}{1-\widetilde{\beta}_{11} S_{1}(0)} \\
& \widetilde{\mathcal{I}}_{2}=\widetilde{\mathcal{I}}_{2}\left(\widetilde{\beta}_{11}\right)=\sum_{i=1}^{\infty} p_{i}\left(\prod_{r=1}^{i-1} \frac{q_{r}}{r v+1}\right)
\end{aligned}
$$

The proof is in Appendix F, where we also give an explicit expression for $\widetilde{\mathcal{I}}_{2}$ in terms of hypergeometric functions.

To compute the attack rate for the vaccination case observe that the change in active cases is given by $I_{i}^{\prime}$, and the change in recovered cases is $I_{i}, i=1,2$; the change in infected is then $I^{\prime}+I$, and the attack rate is

$$
\begin{aligned}
A\left(\widetilde{\beta}_{11}\right) & =\frac{1}{\left.S_{1}(0)+S_{2}(0)\right)} \int_{0}^{\infty}\left(I_{1}^{\prime}+I_{1}+I_{2}^{\prime}+I_{2}\right) d t \\
& =\frac{1}{\left.S_{1}(0)+S_{2}(0)\right)}\left(-I_{1}(0)+\widetilde{\mathcal{I}}_{1}-I_{2}(0)+\widetilde{\mathcal{I}}_{2}\right)
\end{aligned}
$$

The attack rate $A$ is divergent as $\widetilde{\beta}_{11}$ approaches $\widetilde{\beta}_{11}^{*}=1 / S_{1}(0)$, see $(33)$, which is an indication that the linear approximation breaks down, and that this value is likely to be the critical point.

In order to contain the increase in the total cases by no more than a proportion $\epsilon$ we need $\widetilde{\beta}_{11}$ satisfying

$$
A\left(\widetilde{\beta}_{11}\right) \leq A(0)(1+\epsilon)
$$

\section{A.13 Estimate of the peak time during vaccination}

A further evidence of the critical point is obtained by an estimate of the peak time of the infection from (29). Assuming that the active cases in the two subpopulations peak at approximately the same time $\bar{t}$, we set $I_{1}^{\prime}(t)=I_{2}^{\prime}(t)=0$. The solution is

$$
\bar{t}=\frac{1}{v} \log \frac{-\widetilde{\beta}_{12} S_{1}(0) \widetilde{\beta}_{21} S_{2}(0)+\widetilde{\beta}_{22} S_{2}(0)\left(1-\widetilde{\beta}_{11}\right) S_{1}(0)}{1-\widetilde{\beta}_{11} S_{1}(0)} .
$$

Hence, the peak time also diverges at $\widetilde{\beta}_{11}=\widetilde{\beta}_{11}^{*}$.

\section{A.14 Simulations of the phase transition during vaccina- tion}

With the realistic values of the parameters used previously,

$$
S_{1}(0)=0.198872, S_{2}(0)=0.794451, I_{1}(0)=1.97281 \times 10^{-5}, I_{2}(0)=9.55298 \times 10^{-5}
$$

and

we have

$$
\beta_{12}=\widetilde{\beta}_{21}=0.5, \widetilde{\beta}_{22}=2, v=0.1
$$

$$
\widetilde{\beta}_{11}^{*}=1 / S_{1}(0)=5.02836 .
$$

Figure 1 for $t>18$ illustrates a simulation of the differential system, where it is seen that $\widetilde{\beta}_{11}^{*}=5.02836$ is the critical point for the influence of school opening on the overall epidemic.

To achieve a sensible containment take $\epsilon=0.3$, in which case (36) gives $\widetilde{\beta}_{11}<$ 3.03111, a bound also visible in Figure 1 for $t>18$. 


\section{A.15 A SPIAR model}

To illustrate how a phase transition mechanism also appears in more elaborate and realistic models, we develop and simulate one example.

We introduce the compartments of susceptibles $S_{i}$ (not subjected to any virus transmission), presymptomatic $P_{i}$ (infected in incubation period), asymptomatic $A_{i}$ (infected not showing symptoms after incubation), infected $I_{i}$ and recovered $R_{i}$ for $i=1,2$ corresponding to the two subpopulations. A corresponding system could read as follows:

$$
\begin{aligned}
\frac{d S_{1}}{d t} & =-S_{1}\left(\beta_{11}\left(P_{1}+s A_{1}\right)+\beta_{12}\left(P_{2}+A_{2}+\xi I_{2}\right)\right) \\
\frac{d P_{1}}{d t} & =S_{1}\left(\beta_{11}\left(P_{1}+s A_{1}\right)+\beta_{12}\left(P_{2}+A_{2}+\xi I_{2}\right)\right)-\kappa P_{1} \\
\frac{d I_{1}}{d t} & =\epsilon_{1} \kappa P_{1}-I_{1} \\
\frac{d A_{1}}{d t} & =\left(1-\epsilon_{1}\right) \kappa P_{1}-A_{1} \\
\frac{d R_{1}}{d t} & =\left(I_{1}+A_{1}\right) \\
\frac{d S_{2}}{d t} & =-S_{2}\left(\beta_{21}\left(P_{1}+A_{1}+\xi I_{1}\right)+\beta_{22}\left(I_{2}+P_{2}+A_{2}\right)-v\right) \\
\frac{d P_{2}}{d t} & =S_{2}\left(\beta_{21}\left(P_{1}+A_{1}+\xi I_{1}\right)+\beta_{22}\left(I_{2}+P_{2}+A_{2}\right)\right)-\kappa P_{2} \\
\frac{d I_{2}}{d t} & =\epsilon_{2} \kappa P_{2}-I_{2} \\
\frac{d A_{2}}{d t} & =\left(1-\epsilon_{2}\right) \kappa P_{2}-A_{2} \\
\frac{d R_{2}}{d t} & =\left(I_{2}+A_{2}\right)+v S_{2}
\end{aligned}
$$

where the parameters $s, \xi$ represent the fractions of asymptomatic encountered at school and of undetected infected individuals, respectively; $\epsilon_{1}, \epsilon_{2}$ are the fractions of symptomatic in subclass 1 and subclass 2 , respectively; $\kappa$ the rate of exit from latency period. The recovery rate $\gamma$ is normalized to 1 as before, and $v=0$ if there is no ongoing vaccination.

Parameters have been calibrated as given in Table 1 following standard estimations appearing in literature and data studies [36-38].

Figure 11 shows how phase transitions appear also in the SPIAR model. Here, the active cases are given by the sum $P(t)+I(t)+A(t):=P_{1}(t)+I_{1}(t)+A_{1}(t)+P_{2}(t)+$ $I_{2}(t)+A_{2}(t)$, and three scenarios are considered, as before: outbreak, lockdown, and vaccination. In Figure 11, the cyan curve corresponds to closed schools, while the green one is a subcritical pattern; the red curves, instead, show the risk that the pandemic spirals out of control because of insufficiently controlled school opening.

\section{A.16 Other case studies}

A survey of many detailed, data driven studies related to the effect of school opening during the pandemic shows traces of phases transition in all of them. 
Table 1: Recap of the model parameters and their selected values for each scenario in SPIAR model.

\begin{tabular}{lrcc} 
Parameter & Outbreak & Lockdown & Vaccination \\
\hline$\beta_{22}$ & 2 & 0.5 & 1.5 \\
$\beta_{12}=\beta_{21}$ & 0.25 & 0.1 & 0.25 \\
$s$ & 0.9 & 0.9 & 0.9 \\
$\xi$ & 0.3 & 0.3 & 0.3 \\
$k$ & 1 & 1 & 1 \\
$\epsilon_{1}$ & 0.1 & 0.1 & 0.1 \\
$\epsilon_{2}$ & 0.3 & 0.3 & 0.3 \\
$v$ & 0 & 0 & 0.2 \\
\hline
\end{tabular}

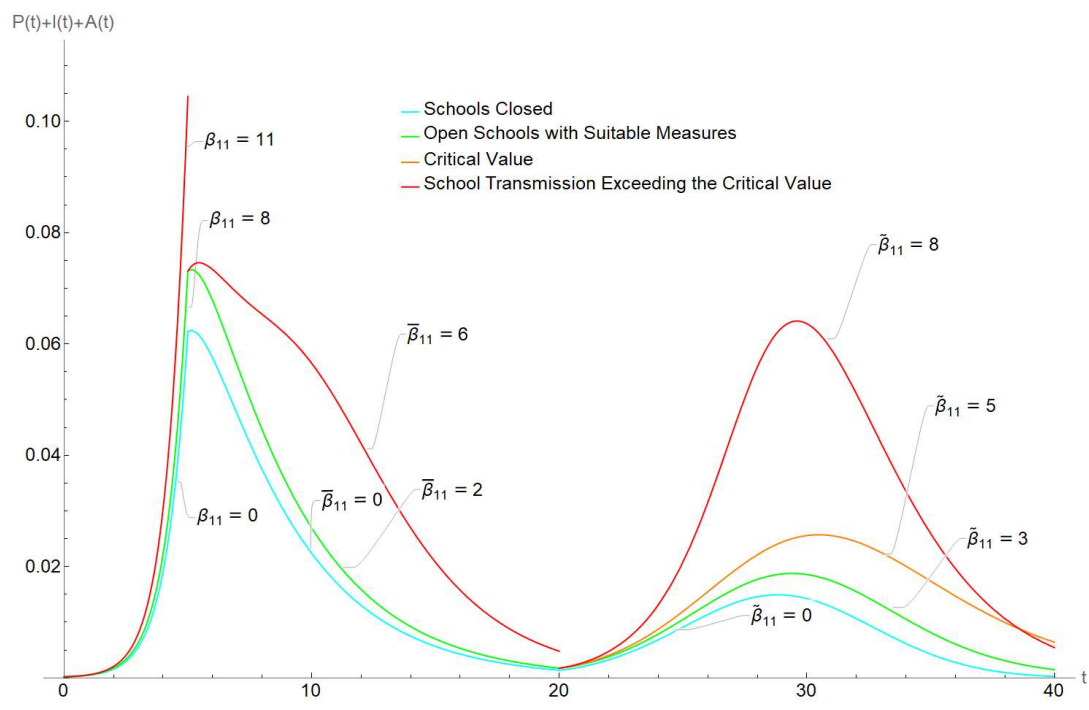

Figure 11: Daily cases for the three scenarios of outbreak, lockdown, and vaccination in an SPIAR model. In each case, there is critical values for the in-school transmission rate $\beta_{11}$, as for SIR model. Cyan curve is with closed schools, green for safe opening, orange for critical values, red for values above criticality. 
[33] uses a detailed compartmental model calibrated on mortality and other estimated and observed data in Bogotà, Colombia, during the whole 2020. The study develops various scenarios of school reopening, and evaluates its impact; the phase transition described in our work appears clearly in Figures 4 and 5 of [33]: one can see that up to $50 \%$ capacity the effect of opening schools is almost negligible, while it becomes substantial above $75 \%$ capacity. This leads to the conclusion that there has to be a critical point between these values.

The appearance of a phase transition phenomenon in [9] has been discussed at length in Section 2.5.

[31] uses a detailed compartmental model and data from the second semester 2020 in Toronto, an outbreak context, to estimate the likely impact of school opening, also discussed in Section 2.5.

[32] analyses French data for late Spring 2020 in order to predict the effect of various forms of school reopening after the end of the lockdown; the paper only makes predictions for short periods (see Figures 3 and 5), and the exact details of the contacts and transmission rates, which are partially estimated and partly obtained from previous measurements, are not provided; still, one can see, especially in Figure 5, that the transmission rates are supercritical, and school opening determines a sharp increase in the overall epidemic spreading.

Among the statistical papers, [24] performs a Time-series analyses using the Bayesian method on Japanese data collected during the initial lockdown, and suggests that school closure did not appear to decrease the incidence of COVID-19. [25], on the other hand, uses US data from the early stages of the outbreak, and regression analysis; the study finds empirical evidence suggesting that school closings dropped infection rate to half: we can interpret this as a sign that transmission rate in schools was supercritical at that time.

An analysis of data gathered by a surveillance of COVID-19 cases in students and staff after reopening of schools across England showed that in-school infections were much less influential than external ones [26]; a study of Italian data from early Fall 2020, a period of low epidemic incidence, also showed very little transmission taking place in schools [27]. These were typical examples of subcritical in-school transmission rate, probably due to the segmented or very controlled reopening of schools.

\section{B Proof of Lemma A.1}

Proof. Consider, first,

$$
\vec{H}=e^{t} \vec{I}
$$

Then

$$
\frac{d \vec{H}}{d t}=e^{t} \vec{I}+e^{t}(A-\mathrm{Id}) \vec{I}=e^{t} A \vec{I}=A e^{t} \vec{I}=A \vec{H}
$$

with $\vec{H}(0)=\vec{I}(0):=\vec{I}_{0}$. Since

$$
\vec{H}=e^{A t} \vec{H}_{0}=e^{A t} \vec{I}_{0}
$$

we get that

$$
\vec{I}=e^{-t} e^{A t} \vec{I}_{0}
$$

We, now, show that

$$
\vec{H}(t)=e^{\lambda_{\max } t} \vec{W}+e^{\lambda_{\min } t} \vec{V}
$$


where $\vec{W}>0$. Then, the assertion of the theorem, which is related to $\vec{I}$, follows immediately from (39).

The rate of growth in the initial exponential phase depends on the largest eigenvalue of the reproduction matrix (12). The result is an immediate consequence of the Perron Frobenius theorem. In fact, since all the elements of $A$ are positive, then the eigenvector $\vec{\xi}$ associated to $\lambda_{\max }$ has positive components while the eigenvector $\vec{\eta}$ associated to $\lambda_{\min }$ has at least one negative component. Since

$$
\vec{H}(t)=e^{\lambda_{\max } t}\left[\begin{array}{l}
\frac{1}{\alpha}\left(\eta_{2} \xi_{1} I_{1}(0)-\eta_{1} \xi_{1} I_{2}(0)\right) \\
\frac{1}{\alpha}\left(\eta_{2} \xi_{2} I_{1}(0)-\eta_{1} \xi_{2} I_{2}(0)\right)
\end{array}\right]+e^{\lambda_{\min } t}\left[\begin{array}{l}
\frac{1}{\alpha}\left(-\eta_{1} \xi_{2} I_{1}(0)+\eta_{1} \xi_{1} I_{2}(0)\right) \\
\frac{1}{\alpha}\left(-\eta_{2} \xi_{2} I_{1}(0)+\eta_{2} \xi_{1} I_{2}(0)\right)
\end{array}\right]
$$

where

$$
\alpha=\operatorname{det}\left[\begin{array}{ll}
\xi_{1} & \eta_{1} \\
\xi_{2} & \eta_{2}
\end{array}\right]=\xi_{1} \eta_{2}-\xi_{2} \eta_{1} .
$$

Then, if, for example, $\eta_{1}<0$ we have $\eta_{2} \geq 0$ hence $\alpha>0$, that is

$$
\begin{aligned}
W_{1} & =\frac{1}{\alpha}\left(\eta_{2} \xi_{1} I_{1,0}-\eta_{1} \xi_{1} I_{2,0}\right)>0 \\
W_{2} & =\frac{1}{\alpha}\left(\eta_{2} \xi_{2} I_{1,0}-\eta_{1} \xi_{2} I_{2,0}\right)>0 .
\end{aligned}
$$

Analogously if $\eta_{1} \geq 0$, then $\eta_{2}<0$, hence $\alpha<0$ and again $W_{1}>0$ and $W_{2}>0$.

\section{Proof of Theorem A.2}

Proof. Note first that

$$
\begin{aligned}
\lambda_{\text {max }, \text { min }}=\frac{\operatorname{tr}(A) \pm \sqrt{(\operatorname{tr}(A))^{2}-4 \operatorname{det}(A)}}{2} & =\frac{a_{11}+a_{22} \pm \sqrt{\left(a_{11}-a_{22}\right)^{2}+4 a_{12} a_{21}}}{2} \\
& >\frac{a_{11}+a_{22} \pm\left(a_{11}-a_{22}\right)}{2}>0,
\end{aligned}
$$

since all the entries of the matrix $A$ are positive. Therefore $\lambda_{\max }, \lambda_{\min }>0$. Furthermore

$$
\lambda^{\prime}\left(a_{11}\right)=\frac{1}{2}\left(1+\frac{a_{11}-a_{22}}{\sqrt{\left(a_{11}-a_{22}\right)^{2}+4 a_{21} a_{12}}}\right)>0
$$

and

We have

$$
\lambda^{\prime \prime}\left(a_{11}\right)=\frac{2 a_{12} a_{21}}{\left(\left(a_{11}-a_{22}\right)^{2}+4 a_{21} a_{12}\right)^{3 / 2}}>0 .
$$

$$
\lambda_{\max }^{\prime}(0)=\frac{1}{2}\left(1-\frac{a_{22}}{\sqrt{a_{22}^{2}+4 a_{12} a_{21}}}\right)
$$

and

$$
\begin{aligned}
\lambda_{\text {max }}^{\prime}\left(a_{22}-\alpha \sqrt{a_{12} a_{21}}\right) & =\frac{1}{2}\left(1-\frac{\alpha \sqrt{a_{12} a_{21}}}{\sqrt{\alpha^{2} a_{12} a_{21}+4 a_{12} a_{21}}}\right) \\
& =\frac{1}{2}\left(1-\frac{\alpha \sqrt{a_{12} a_{21}}}{\sqrt{\alpha^{2}+4} \sqrt{a_{12} a_{21}}}\right)=\frac{1}{2}\left(1-\frac{\alpha}{\sqrt{\alpha^{2}+4}}\right) .
\end{aligned}
$$


Analogously

$$
\lambda_{\max }^{\prime}\left(a_{22}+\alpha \sqrt{a_{12} a_{21}}\right)=\frac{1}{2}\left(1+\frac{\alpha}{\sqrt{\alpha^{2}+4}}\right) .
$$

Finally

$$
\lambda_{\max }^{\prime}\left(2 a_{22}\right)=\frac{1}{2}\left(1+\frac{a_{22}}{\sqrt{a_{22}^{2}+4 a_{12} a_{21}}}\right) .
$$

Observe now that

$$
\begin{aligned}
\lambda_{\max }^{\prime}\left(a_{22}-\alpha \sqrt{a_{12} a_{21}}\right)-\lambda_{\max }^{\prime}(0) & =\frac{1}{2}\left(1-\frac{\alpha}{\sqrt{\alpha^{2}+4}}\right)-\frac{1}{2}\left(1-\frac{a_{22}}{\sqrt{a_{22}^{2}+4 a_{12} a_{21}}}\right) \\
& -\frac{\alpha}{2 \sqrt{\alpha^{2}+4}}+\frac{a_{22}}{2 \sqrt{a_{22}^{2}+4 a_{12} a_{21}}} \\
& =\lambda_{\max }^{\prime}\left(2 a_{22}\right)-\lambda_{\max }^{\prime}\left(a_{22}+\alpha \sqrt{a_{12} a_{21}}\right) .
\end{aligned}
$$

Also note that

$$
\lambda_{\max }^{\prime}\left(a_{22}+\alpha \sqrt{a_{12} a_{21}}\right)-\lambda_{\max }^{\prime}\left(a_{22}-\alpha \sqrt{a_{12} a_{21}}\right)=\frac{\alpha}{\sqrt{4+\alpha^{2}}} .
$$

Then

$$
\begin{aligned}
& \frac{\lambda_{\max }^{\prime}\left(a_{22}+\alpha \sqrt{a_{12} a_{21}}\right)-\lambda_{\max }^{\prime}\left(a_{22}-\alpha \sqrt{a_{12} a_{21}}\right)}{\lambda_{\max }^{\prime}\left(a_{22}-\alpha \sqrt{a_{12} a_{21}}\right)-\lambda_{\max }^{\prime}(0)} \\
& =\frac{\lambda_{\max }^{\prime}\left(a_{22}+\alpha \sqrt{a_{12} a_{21}}\right)-\lambda_{\max }^{\prime}\left(a_{22}-\alpha \sqrt{a_{12} a_{21}}\right)}{\lambda_{\max }^{\prime}\left(2 a_{22}\right)-\lambda_{\max }^{\prime}\left(a_{22}+\alpha \sqrt{a_{12} a_{21}}\right)}=\frac{\frac{\alpha}{\sqrt{4+\alpha^{2}}}}{-\frac{\alpha}{2 \sqrt{4+\alpha^{2}}}+\frac{a_{22}}{2 \sqrt{a_{22}^{2}+4 a_{12} a_{21}}}} \\
& =\frac{2 \alpha \sqrt{a_{22}^{2}+4 a_{12} a_{21}}}{a_{22} \sqrt{\alpha^{2}+4}-\alpha \sqrt{a_{22}^{2}+4 a_{12} a_{21}}} \geq \frac{2 \alpha}{\sqrt{\alpha^{2}+4}-\alpha} .
\end{aligned}
$$

Since $\sqrt{a_{22}^{2}+4 a_{12} a_{21}}>a_{22}$ and

$$
a_{22} \sqrt{\alpha^{2}+4}-\alpha \sqrt{a_{22}^{2}+4 a_{12} a_{21}} \leq a_{22} \sqrt{\alpha^{2}+4}-\alpha a_{22}
$$

and the claim follows.

\section{Proof of Corollary A.3}

Proof. We show the result for $\vec{H}$, which is defined in (38). Then, the result for $\vec{I}$ follows straightforwardly using $(\underset{\sim}{39})$.

Note that $\lambda_{2}>\lambda_{1}>\lambda_{0}$ and $\tilde{\lambda_{2}}>\tilde{\lambda}_{1}>\tilde{\lambda_{0}}$. We proceed componentwise. Since $V_{1}^{0}>0, V_{1}^{2}>0$

$$
\begin{aligned}
\left|H_{1}^{\lambda_{2}}(t)-H_{1}^{\lambda_{0}}(t)\right| & =\left|e^{\lambda_{2} t} W_{1}^{2}+e^{\widetilde{\lambda_{2}} t} V_{1}^{2}-e^{\lambda_{0} t} W_{1}^{0}-e^{\widetilde{\lambda_{0}} t} V_{1}^{0}\right| \\
& >e^{\lambda_{2} t} W_{1}^{2}\left|1-\frac{W_{1}^{0}}{W_{1}^{2}} e^{\left(\lambda_{0}-\lambda_{2}\right) t}-\frac{V_{1}^{0}}{W_{1}^{2}} e^{\left(\widetilde{\lambda_{0}}-\lambda_{2}\right) t}\right|
\end{aligned}
$$

and noticing that

$$
\begin{aligned}
& \lambda_{0}-\lambda_{2}<-\sqrt{2} \sqrt{a_{21} a_{12}} \\
& \widetilde{\lambda_{0}}-\lambda_{2}<\widetilde{\lambda_{2}}-\lambda_{2}=-2 \sqrt{2} \sqrt{a_{21} a_{12}}
\end{aligned}
$$


we get

$$
\left|H_{1}^{\lambda_{2}}(t)-H_{1}^{\lambda_{0}}(t)\right|>e^{\lambda_{2} t} W_{1}^{2}\left|1-\left(\frac{W_{1}^{0}}{W_{1}^{2}}+\frac{V_{1}^{0}}{W_{1}^{2}}\right) e^{-\sqrt{2 a_{12} a_{21}} t}\right|
$$

and setting $\frac{W_{1}^{0}+V_{1}^{0}}{W_{1}^{2}}=: Q_{1}$ we can pick up $t$ such that $1-Q_{1} e^{-\sqrt{2 a_{12} a_{21}} t}>\frac{1}{2}$ that is $e^{\sqrt{2 a_{12} a_{21}} t}>2 Q_{1}$ hence $t>\frac{1}{\sqrt{2 a_{12} a_{21}}} \ln \left(2 Q_{1}\right)=: t_{1}^{0}$ so that finally

$$
\left|H_{1}^{\lambda_{2}}(t)-H_{1}^{\lambda_{0}}(t)\right|>\frac{1}{2} W_{1} e^{\lambda_{2} t} .
$$

On the other hand

$$
\begin{aligned}
k\left|H_{1}^{\lambda_{1}}(t)-H_{1}^{\lambda_{0}}(t)\right| & =k\left|e^{\lambda_{1} t} W_{1}^{1}+e^{\widetilde{\lambda_{1}} t} V_{1}^{1}-e^{\lambda_{0} t} W_{1}^{0}-e^{\widetilde{\lambda_{0}} t} V_{1}^{0}\right| \\
& \leq k e^{\lambda_{1} t} W_{1}^{1}\left(1+e^{\left(\widetilde{\lambda_{1}}-\lambda_{1}\right)} \frac{V_{1}^{1}}{W_{1}^{1}}+e^{\left(\lambda_{0}-\lambda_{1}\right) t} \frac{W_{1}^{0}}{W_{1}^{1}}+e^{\left(\widetilde{\lambda_{0}}-\lambda_{1}\right) t} \frac{V_{1}^{0}}{W_{1}^{1}}\right) .
\end{aligned}
$$

Using the fact that $\widetilde{\lambda_{1}}-\lambda_{1}<0, \lambda_{0}-\lambda_{1}<0$ and $\widetilde{\lambda_{0}}-\lambda_{1}<\widetilde{\lambda_{1}}-\lambda_{1}<0$, from (42) we get

$$
k\left|H_{1}^{\lambda_{1}}(t)-H_{1}^{\lambda_{0}}(t)\right| \leq k e^{\lambda_{1} t} W_{1}^{1}\left(1+\frac{V_{1}^{1}+W_{1}^{0}+V_{1}^{0}}{W_{1}^{1}}\right)=k W_{1} e^{\lambda_{1} t}\left(1+Q_{1}+\frac{V_{1}^{1}}{W_{1}^{1}}\right) .
$$

Hence, by (41) and (43) we then get

$$
\frac{1}{2} W_{1} e^{\lambda_{2} t}>k W_{1} e^{\lambda_{1} t}\left(1+Q_{1}+\frac{V_{1}^{1}}{W_{1}^{1}}\right)
$$

that is

hence

$$
\begin{aligned}
& e^{\left(\lambda_{2}-\lambda_{1}\right) t}>2 k\left(1+Q_{1}+\frac{V_{1}^{1}}{W_{1}^{1}}\right) \\
& e^{2 \sqrt{a_{12} a_{21}} t}>2 k\left(1+Q_{1}+\frac{V_{1}^{1}}{W_{1}^{1}}\right)
\end{aligned}
$$

$$
t>\frac{1}{2 \sqrt{a_{12} a_{21}}} \ln \left(2 k\left(1+Q_{1}+\frac{V_{1}^{1}}{W_{1}^{1}}\right)\right)=: t_{1}^{1} .
$$

Therefore, for $t \geq \max \left(t_{1}^{0}, t_{1}^{1}\right)$

$$
\left|H_{1}^{\lambda_{2}}(t)-H_{1}^{\lambda_{0}}(t)\right|>k\left|H_{1}^{\lambda_{1}}(t)-H_{1}^{\lambda_{0}}(t)\right| .
$$

Analogously one can show that

$$
\left|H_{2}^{\lambda_{2}}(t)-H_{2}^{\lambda_{0}}(t)\right| \geq k\left|H_{2}^{\lambda_{1}}(t)-H_{2}^{\lambda_{0}}(t)\right|
$$

for $t \geq \max \left(t_{2}^{0}, t_{2}^{1}\right)$, where

$$
\begin{aligned}
t_{2}^{0} & =\frac{1}{\sqrt{2 a_{21} a_{12}}} \ln \left(2 Q_{2}\right), \\
Q_{2} & =\frac{W_{2}^{0}+V_{2}^{0}}{W_{2}^{2}} \\
t_{2}^{1} & =\frac{1}{2 \sqrt{a_{12} a_{21}}} \ln \left(2 k\left(1+Q_{2}+\frac{V_{2}^{2}}{W_{2}^{2}}\right)\right) .
\end{aligned}
$$

So picking up $t>\max \left(t_{1}^{0}, t_{1}^{1}, t_{2}^{0}, t_{2}^{1}\right)$ and using (39), the claim follows. 


\section{E An application of Theorem A.2}

Inequality (14) indicates a phase transition. In fact from now on let $a_{22}^{2}>>a_{12} a_{21}$ and $\alpha=2$. In this case

$$
\frac{2 \alpha}{\sqrt{\alpha^{2}+4}-\alpha} \simeq 4.8
$$

and so by (14) we have

$$
\Delta_{1} \geq 4.8 \Delta_{0}=4.8 \Delta_{2}
$$

Given

and

$$
\lambda_{\max }^{\prime}(0)=\frac{1}{2}\left(1-\frac{a_{22}}{\sqrt{a_{22}^{2}+4 a_{12} a_{21}}}\right)
$$

$$
\lambda_{\text {max }}^{\prime}\left(a_{22}-\alpha \sqrt{a_{12} a_{21}}\right)=\frac{1}{2}\left(1-\frac{\alpha}{\sqrt{\alpha^{2}+4}}\right)
$$

if $\lambda_{\max }^{\prime}(0)<<\lambda_{\max }^{\prime}\left(a_{22}-\alpha \sqrt{a_{12} a_{21}}\right) / 4.8$ then from (14)

$$
\begin{aligned}
\lambda_{\max }^{\prime}\left(a_{22}+\alpha \sqrt{a_{12} a_{21}}\right) & \geq 5.8 \lambda_{\max }^{\prime}\left(a_{22}-\alpha \sqrt{a_{12} a_{21}}\right)-4.8 \lambda_{\max }^{\prime}(0) \\
& \geq 4.8 \lambda_{\max }^{\prime}\left(a_{22}-\alpha \sqrt{a_{12} a_{21}}\right) .
\end{aligned}
$$

Hence, by (44) and since $\lambda_{\max }^{\prime}$ is increasing

$$
\begin{aligned}
4.8\left(\lambda_{\max }\left(a_{22}-\alpha \sqrt{a_{12} a_{21}}\right)-\lambda_{\max }(0)\right) & \leq 4.8 \lambda_{\max }^{\prime}\left(a_{22}-\alpha \sqrt{a_{21} a_{12}}\right)\left(a_{22}-\alpha \sqrt{a_{12} a_{21}}\right) \\
& \leq \lambda_{\max }^{\prime}\left(a_{22}+\alpha \sqrt{a_{12} a_{21}}\right)\left(2 a_{22}-\left(a_{22}+\alpha \sqrt{a_{12} a_{21}}\right)\right) \\
& \leq \lambda_{\max }\left(2 a_{22}\right)-\lambda_{\max }\left(a_{22}+\alpha \sqrt{a_{21} a_{12}}\right) .
\end{aligned}
$$

Therefore

$$
\lambda_{\max }\left(2 a_{22}\right)-\lambda_{\max }(0) \geq 4.8\left(\lambda_{\max }\left(a_{22}-\alpha \sqrt{a_{21} a_{12}}\right)-\lambda_{\max }(0)\right) .
$$

Hence $\forall 0<a_{11} \leq a_{22}-\alpha \sqrt{a_{12} a_{21}}$, we have

$$
\lambda_{\max }\left(2 a_{22}\right)-\lambda_{\max }(0) \geq 4.8\left(\lambda_{\max }\left(a_{11}\right)-\lambda_{\max }(0)\right) .
$$

Last inequality tells us that closing schools starting from a reproduction number $2 a_{22}$ is much more effective than starting from a reproduction number around $a_{22}$.

\section{F Proof of Theorem A.4}

Proof. Let

$$
\begin{aligned}
& \widetilde{\mathcal{I}}_{1}^{(k)}:=\int_{0}^{+\infty} I_{1}(t) e^{-k v t} d t \\
& \tilde{\mathcal{I}}_{2}^{(k)}:=\int_{0}^{+\infty} I_{2}(t) e^{-k v t} d t .
\end{aligned}
$$

Integrating the first equation in (29), we have

$$
-I_{1}(0)=\left(\widetilde{\beta}_{11} S_{1}(0)-1\right) \widetilde{\mathcal{I}}_{1}^{(0)}+\widetilde{\beta}_{12} S_{1}(0) \widetilde{\mathcal{I}}_{2}^{(0)}
$$


which implies

$$
\widetilde{\mathcal{I}}_{1}^{(0)}=\frac{\widetilde{\beta}_{12} S_{1}(0) \widetilde{\mathcal{I}}_{2}^{(0)}+I_{1}(0)}{1-\widetilde{\beta}_{11} S_{1}(0)}>0 \text { if } \widetilde{\beta}_{11} S_{1}(0)<1 .
$$

This proves (33) (note that $\widetilde{\mathcal{I}}_{1}^{(0)} \equiv \widetilde{\mathcal{I}}_{1}$ ).

Integrating the second equation in $(29)$ we get

$$
-I_{2}(0)=\widetilde{\beta}_{21} S_{2}(0) \widetilde{\mathcal{I}}_{1}^{(1)}+\widetilde{\beta}_{22} S_{2}(0) \widetilde{\mathcal{I}}_{2}^{(1)}-\widetilde{\mathcal{I}}_{2}^{(0)}
$$

which implies

$$
\widetilde{\mathcal{I}}_{2}^{(0)}=\widetilde{\mathcal{I}}_{2}^{(0)}\left(\widetilde{\mathcal{I}}_{1}^{(1)}, \widetilde{\mathcal{I}}_{2}^{(1)}\right)=\widetilde{\beta}_{21} S_{2}(0) \widetilde{\mathcal{I}}_{1}^{(1)}+\widetilde{\beta}_{22} S_{2}(0) \widetilde{\mathcal{I}}_{2}^{(1)}+I_{2}(0) .
$$

Substituting in (48), we get

$$
\widetilde{\mathcal{I}}_{1}^{(0)}=\widetilde{\mathcal{I}}_{1}^{(0)}\left(\widetilde{\mathcal{I}}_{1}^{(1)}, \widetilde{\mathcal{I}}_{2}^{(1)}\right)=\frac{\widetilde{\beta}_{12} S_{1}(0)\left(\widetilde{\beta}_{21} S_{2}(0) \widetilde{\mathcal{I}}_{1}^{(1)}+\widetilde{\beta}_{22} S_{2}(0) \widetilde{\mathcal{I}}_{2}^{(1)}+I_{2}(0)\right)+I_{1}(0)}{1-\widetilde{\beta}_{11} S_{1}(0)}
$$

which in turn implies

$$
\begin{aligned}
\widetilde{\mathcal{I}}_{2}^{(0)} & =\widetilde{\mathcal{I}}_{2}^{(0)}\left(\widetilde{\mathcal{I}}_{2}^{(1)}\right) \\
& =\widetilde{\beta}_{21} S_{2}(0) \widetilde{\mathcal{I}}_{1}^{(1)}+\widetilde{\beta}_{22} S_{2}(0) \widetilde{\mathcal{I}}_{2}^{(1)}+I_{2}(0) \\
& =\widetilde{\beta}_{21} S_{2}(0) \frac{\frac{I_{1}(0)}{v}+\frac{1}{v} \widetilde{\beta}_{12} S_{1}(0) \widetilde{\mathcal{I}}_{2}^{(1)}}{1-\frac{1}{v}\left(\widetilde{\beta}_{11} S_{1}(0)-1\right)}+\widetilde{\beta}_{22} S_{2}(0) \widetilde{\mathcal{I}}_{2}^{(1)}+I_{2}(0) \\
& =\frac{\widetilde{\beta}_{21} S_{2}(0) I_{1}(0)}{v-\left(\widetilde{\beta}_{11} S_{1}(0)-1\right)}+I_{2}(0)+\widetilde{\mathcal{I}}_{2}^{(1)}\left(\widetilde{\beta}_{22} S_{2}(0)+\frac{\widetilde{\beta}_{21} S_{2}(0) \widetilde{\beta}_{12} S_{1}(0)}{v-\left(\widetilde{\beta}_{11} S_{1}(0)-1\right)}\right) \\
& =p_{1}+\widetilde{\mathcal{I}}_{2}^{(1)} q_{1} .
\end{aligned}
$$

For each $k \geq 1$, it follows from (29), and integration by parts that

$$
\begin{aligned}
\widetilde{\mathcal{I}}_{1}^{(k)} & =\int_{0}^{+\infty} I_{1} e^{-k v t} d t \\
& =\frac{I_{1}(0)}{k v}+\int_{0}^{+\infty}\left(\widetilde{\beta}_{11} S_{1}(0) I_{1}+\widetilde{\beta}_{12} S_{1}(0) I_{2}-I_{1}\right) \frac{e^{-k v t}}{k v} d t \\
& =\frac{I_{1}(0)}{k v}+\frac{1}{k v}\left(\left(\widetilde{\beta}_{11} S_{1}(0)-1\right) \widetilde{\mathcal{I}}_{1}^{(k)}+\widetilde{\beta}_{12} S_{1}(0) \widetilde{\mathcal{I}}_{2}^{(k)}\right)
\end{aligned}
$$

which yields

$$
\widetilde{\mathcal{I}}_{1}^{(k)}\left(\widetilde{\mathcal{I}}_{2}^{(k)}\right)=\frac{I_{1}(0)+\widetilde{\beta}_{12} S_{1}(0) \widetilde{\mathcal{I}}_{2}^{(k)}}{k v-\left(\widetilde{\beta}_{11} S_{1}(0)-1\right)}>0
$$

and

$$
\begin{aligned}
\widetilde{\mathcal{I}}_{2}^{(k)} & =\int_{0}^{+\infty} I_{2} e^{-k v t} d t= \\
& =\frac{I_{2}(0)}{k v}+\int_{0}^{+\infty}\left(\widetilde{\beta}_{21} S_{1}(0) e^{-v t} I_{1}+\widetilde{\beta}_{22} S_{1}(0) e^{-v t} I_{2}-I_{2}\right) \frac{e^{-k v t}}{k v} d t \\
& =\frac{1}{k v}\left(I_{2}(0)+\widetilde{\beta}_{21} S_{2}(0) \widetilde{\mathcal{I}}_{1}^{(k+1)}+\widetilde{\beta}_{22} S_{2}(0) \widetilde{\mathcal{I}}_{2}^{(k+1)}-\widetilde{\mathcal{I}}_{2}^{(k)}\right)
\end{aligned}
$$


that gives

$$
\widetilde{\mathcal{I}}_{2}^{(k)}=\frac{1}{(k v+1)}\left(I_{2}(0)+\widetilde{\beta}_{21} S_{1}(0) \widetilde{\mathcal{I}}_{1}^{(k+1)}+\widetilde{\beta}_{22} S_{2}(0) \widetilde{\mathcal{I}}_{2}^{(k+1)}\right) .
$$

This implies

$$
\begin{aligned}
& \widetilde{\mathcal{I}}_{2}^{(k)}\left(\widetilde{\mathcal{I}}_{2}^{(k+1)}\right)= \frac{1}{k v+1}\left(I_{2}(0)+\widetilde{\beta}_{21} S_{2}(0) \frac{I_{1}(0)+\widetilde{\beta}_{12} S_{1}(0) \widetilde{\mathcal{I}}_{2}^{(k+1)}}{(k+1) v-\widetilde{\beta}_{11} S_{1}(0)+1}+\widetilde{\beta}_{22} S_{2}(0) \widetilde{\mathcal{I}}_{2}^{(k+1)}\right) \\
&= \frac{1}{k v+1}\left(I_{2}(0)+\frac{\widetilde{\beta}_{21} S_{2}(0) I_{1}(0)}{(k+1) v-\widetilde{\beta}_{11} S_{1}(0)+1}\right. \\
&\left.\quad+\widetilde{\mathcal{I}}_{2}^{(k+1)}\left(\widetilde{\beta}_{22} S_{2}(0)+\frac{\widetilde{\beta}_{12} \widetilde{\beta}_{21} S_{1}(0) S_{2}(0)}{(k+1) v-\widetilde{\beta}_{11} S_{1}(0)+1}\right)\right) \\
&=\frac{1}{k v+1}\left(p_{k+1}+\widetilde{\mathcal{I}}_{2}^{(k+1)} q_{k+1}\right) .
\end{aligned}
$$

From (52) and (55)

$$
\begin{aligned}
\tilde{\mathcal{I}}_{2}^{(0)} & =p_{1}+q_{1} \tilde{\mathcal{I}}_{2}^{(1)} \\
& =p_{1}+q_{1} \frac{1}{v+1}\left(p_{2}+q_{2} H_{2}\right) \\
& =\ldots \\
& =\sum_{i=1}^{\infty} p_{i}\left(\prod_{r=1}^{i-1} \frac{q_{r}}{r v+1}\right) .
\end{aligned}
$$

This proves (34) (note that $\widetilde{\mathcal{I}}_{2}^{(0)} \equiv \widetilde{\mathcal{I}}_{2}$ ). Using Wolfram Mathematica for symbolic calculations, we provide an explicit expression for $\widetilde{\mathcal{I}}_{2}^{(0)}\left(\widetilde{\beta}_{11}\right)$ in terms of special functions

$$
\begin{aligned}
& \left\{S_{2}(0)\left(1-\widetilde{\beta}_{11} S_{1}(0)+v\right)\right\}^{-1}\left\{\left[I_{2}(0) S_{2}(0)\left(1-\widetilde{\beta}_{11} S_{1}(0)+v\right)\right] \times\right. \\
& \times{ }_{2} F_{2}\left(1,1+\frac{1}{v}-\frac{\widetilde{\beta}_{11} S_{1}(0)}{v}+\frac{\widetilde{\beta}_{12} \widetilde{\beta}_{21} S_{1}(0)}{v \widetilde{\beta}_{22}} ; 1+\frac{1}{v}, 1+\frac{1}{v}-\frac{\widetilde{\beta}_{11} S_{1}(0)}{v} ; \frac{\widetilde{\beta}_{22} S_{2}(0)}{v}\right) \\
& \left.+\widetilde{\beta}_{21} I_{1}(0) S_{2}^{2}(0){ }_{2} F_{2}\left(1,1+\frac{1}{v}-\frac{\widetilde{\beta}_{11} S_{1}(0)}{v}+\frac{\widetilde{\beta}_{12} \widetilde{\beta}_{21} S_{1}(0)}{v \widetilde{\beta}_{22}} ; 1+\frac{1}{v}, 2+\frac{1}{v}-\frac{\widetilde{\beta}_{11} S_{1}(0)}{v} ; \frac{\widetilde{\beta}_{22} S_{2}(0)}{v}\right)\right\}
\end{aligned}
$$

where ${ }_{p} F_{q}(\vec{a} ; \vec{b} ; z)$ is the generalized hypergeometric function, see for example [8].

Contact address: New York University Abu Dhabi, Division of Science, Abu Dhabi, 129188, UAE

Contact e-mail: ag189@nyu.edu 


\section{Figures}

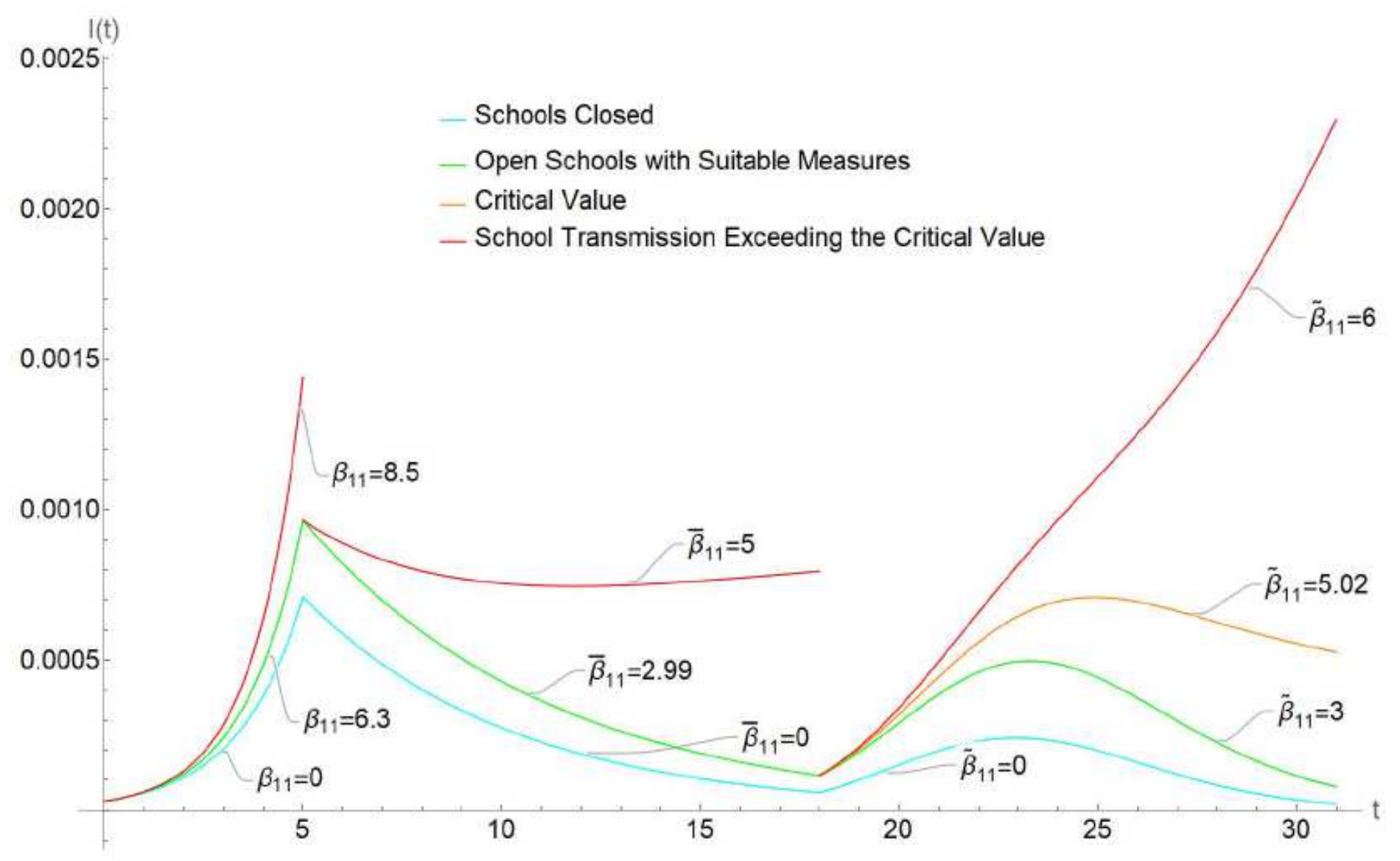

\section{Figure 1}

Daily active cases $11(\mathrm{t})+\mathrm{I} 2(\mathrm{t})$ for various scenarios: outbreak or new strain upsurge, lockdown, and vaccination. In each case, there is critical value for the in-school transmission rate $\beta 11$. Cyan curve is with closed schools, green for safe opening, orange for critical values, red for values above criticality. 


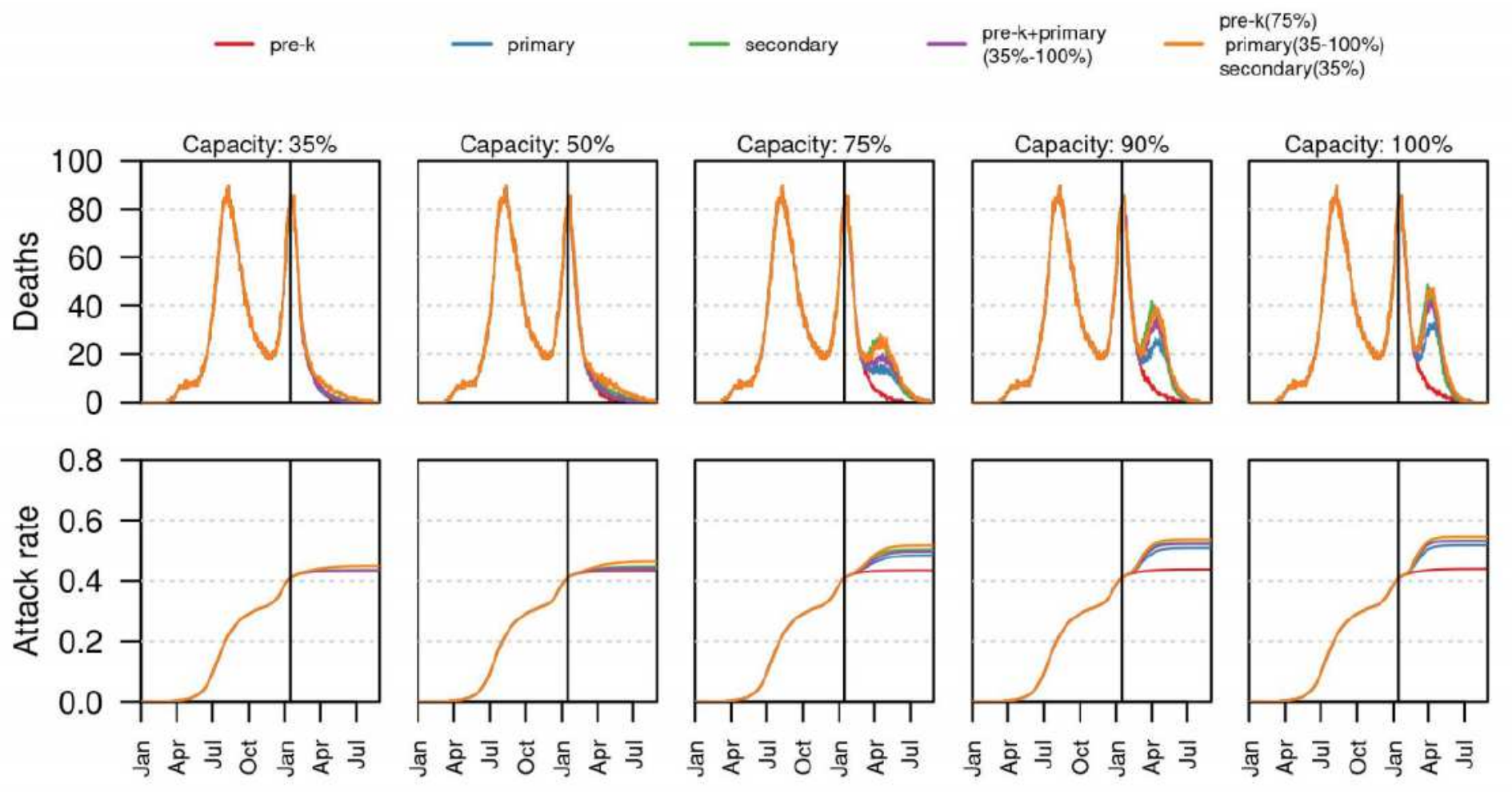

Figure 2

The impact of school reopening strategies in time as simulated in [33] from data from Bogotà, Colombia, for various values of the capacity, i.e. the percentage of students allowed back at school. Each column shows a different capacity level. Top panel shows the median daily incidence of deaths for each reopening strategy based on grades. Bottom panel shows the estimated attack rate for each of the reopening scenarios. Vertical black line shows the timing of school reopening (January 25, 2021). All scenarios were simulated up to August 31, 2021. 


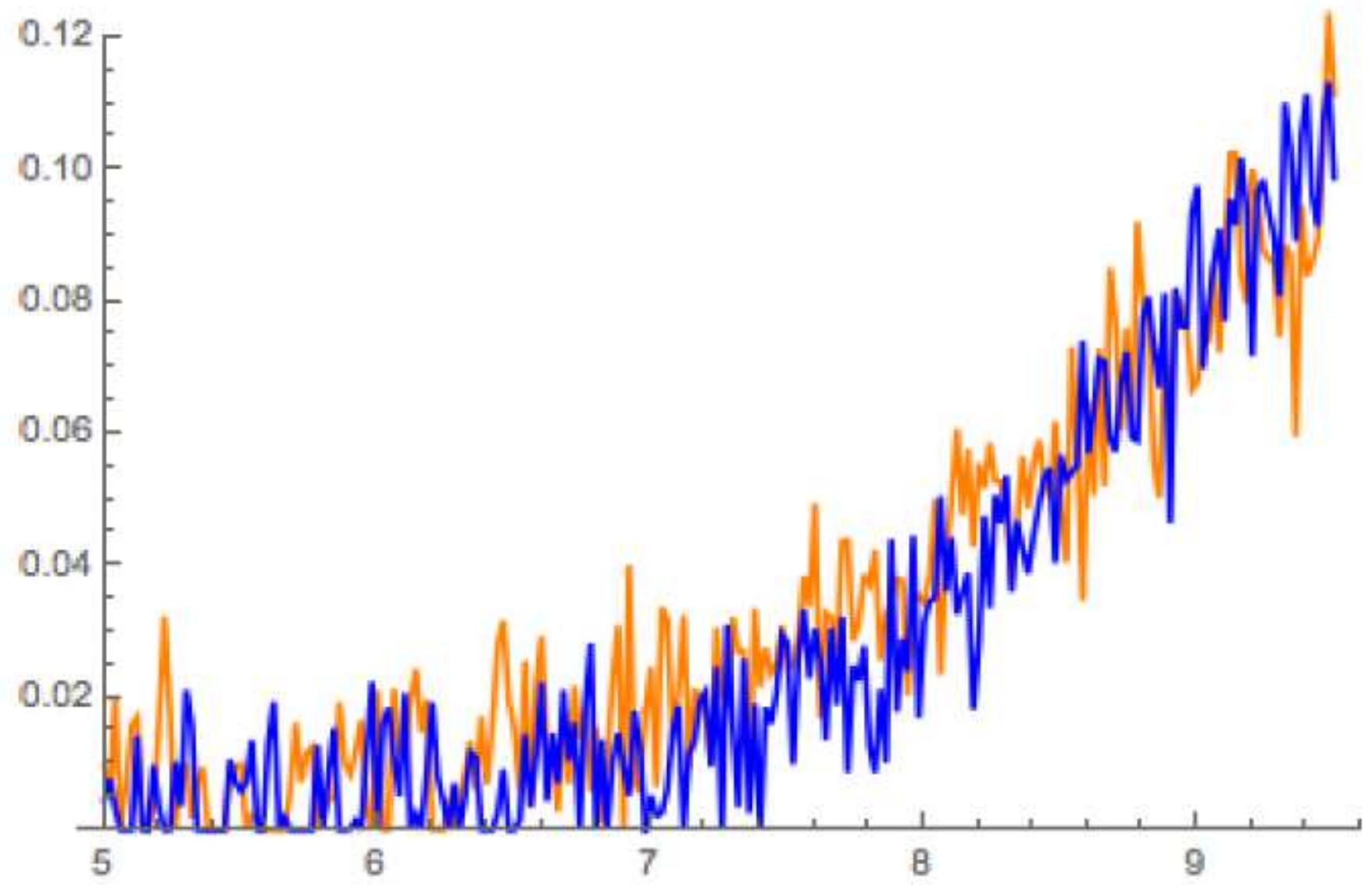

Figure 3

Synthetic data of daily active cases in the two different scenarios, one with subcritical and one with supercritical in-school transmission rates, and slightly different initial number of cases. Gaussian noise has been added to make the example more realistic. 


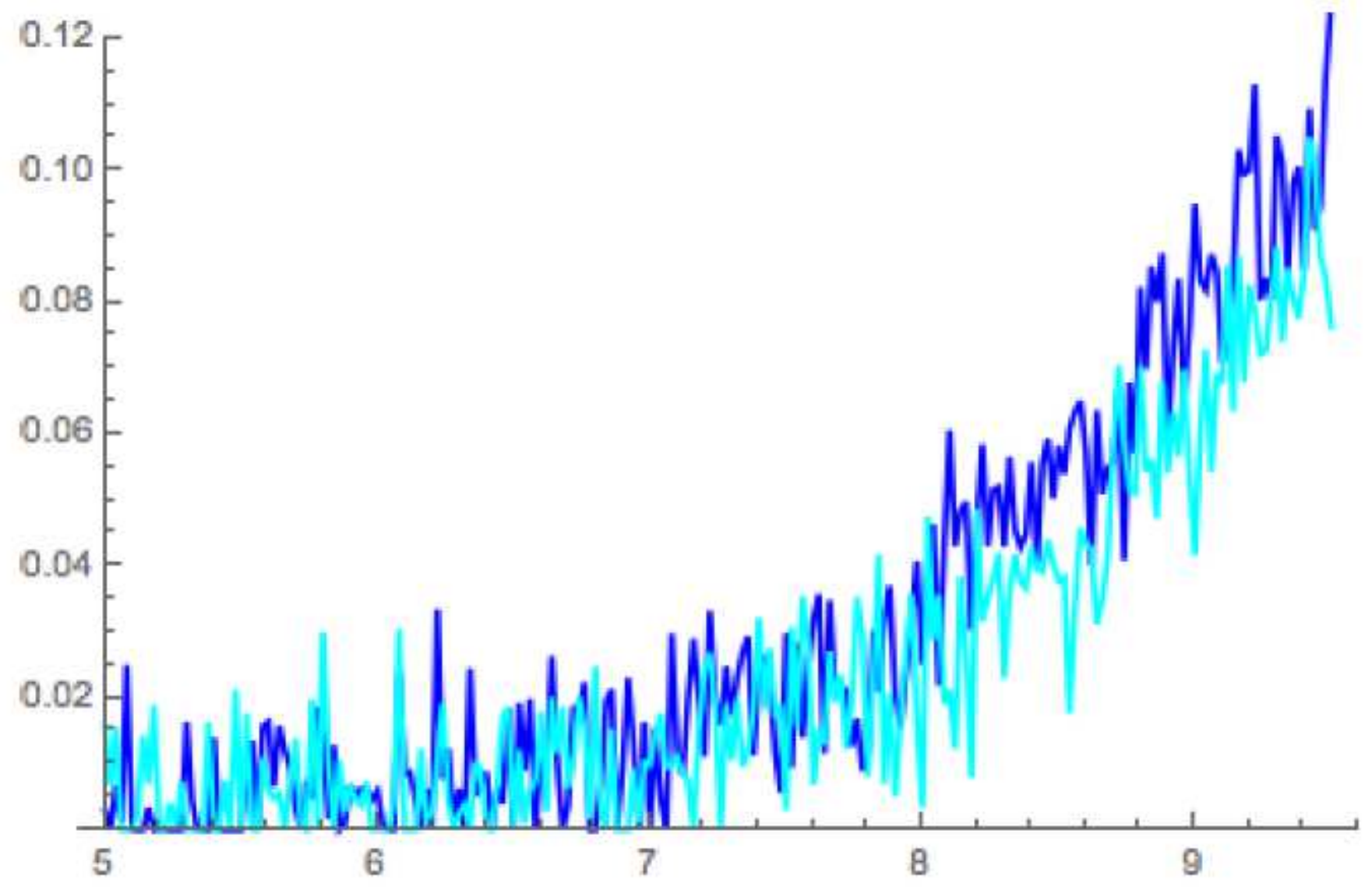

Figure 4

Reduction in daily cases due to school closure in the first scenario 


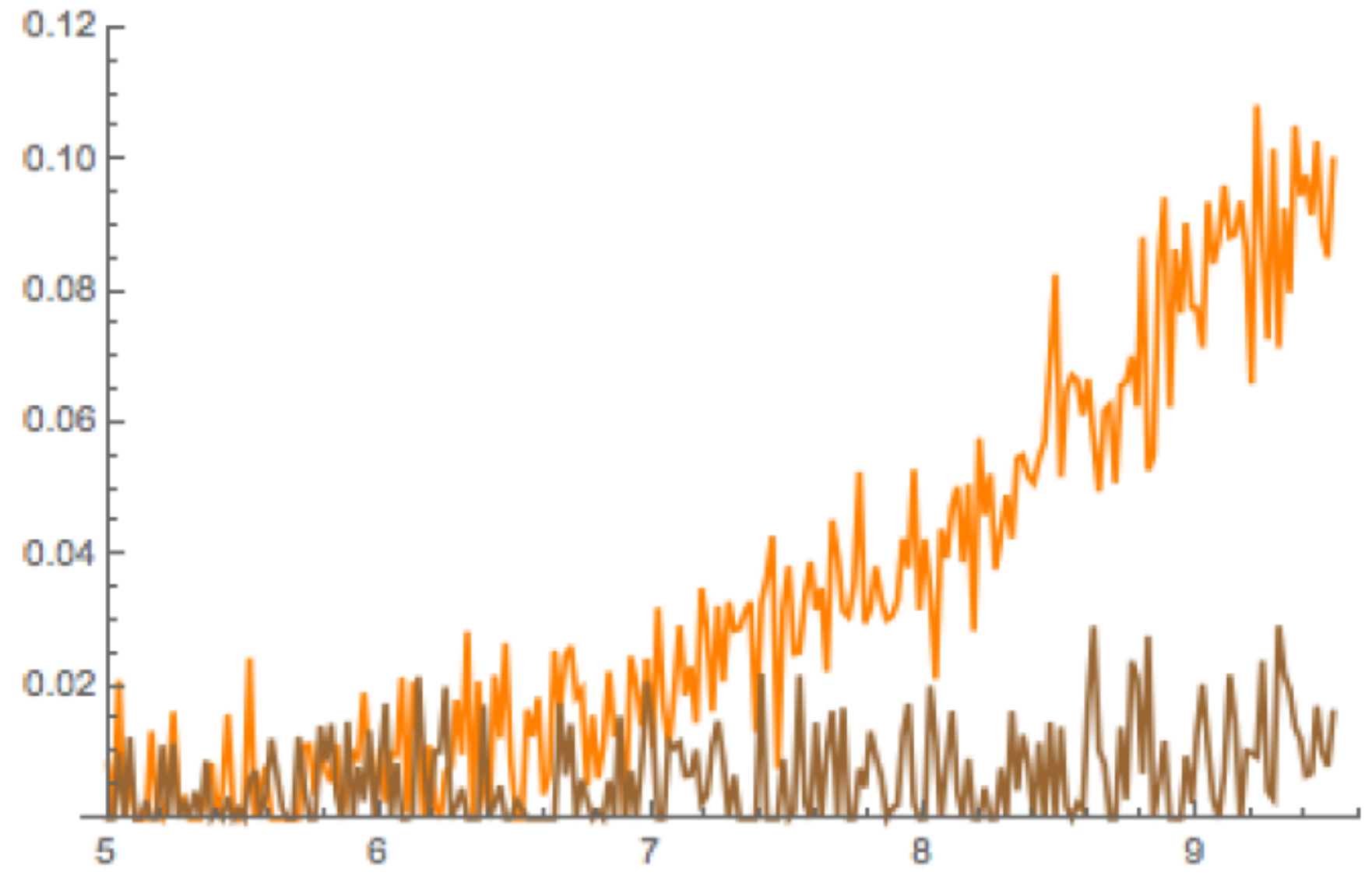

\section{Figure 5}

Reduction in daily cases due to school closure in the second scenario 
$\lambda_{\text {max }}^{\prime}\left(a_{11}\right)$

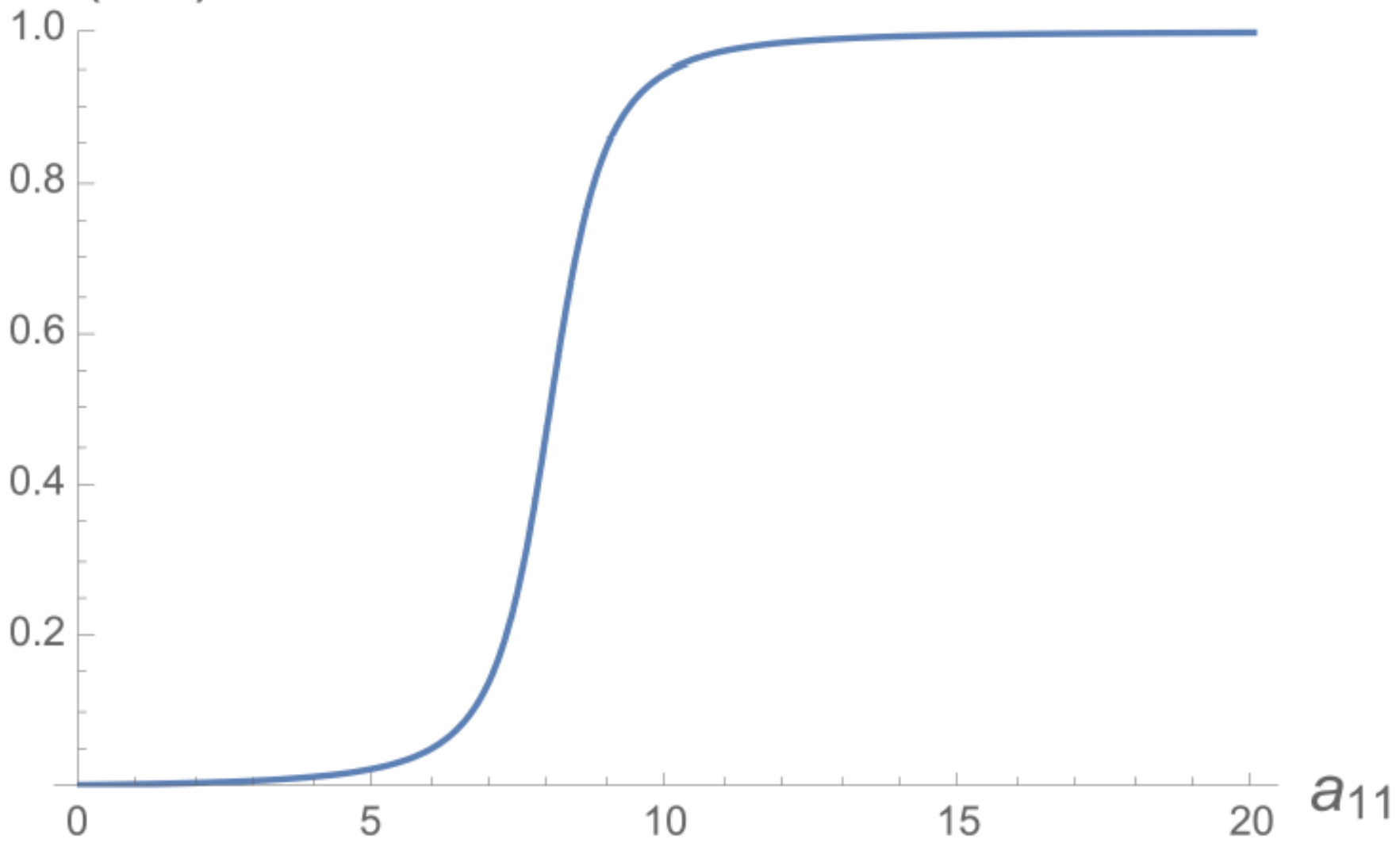

Figure 6

Derivative of the largest eigenvalue of $A=($ aij $)$ with respect to a11, with a12 $=a 21=0.5$ and a22 $=8$. 


\section{$\lambda_{\max }\left(a_{11}\right)$}

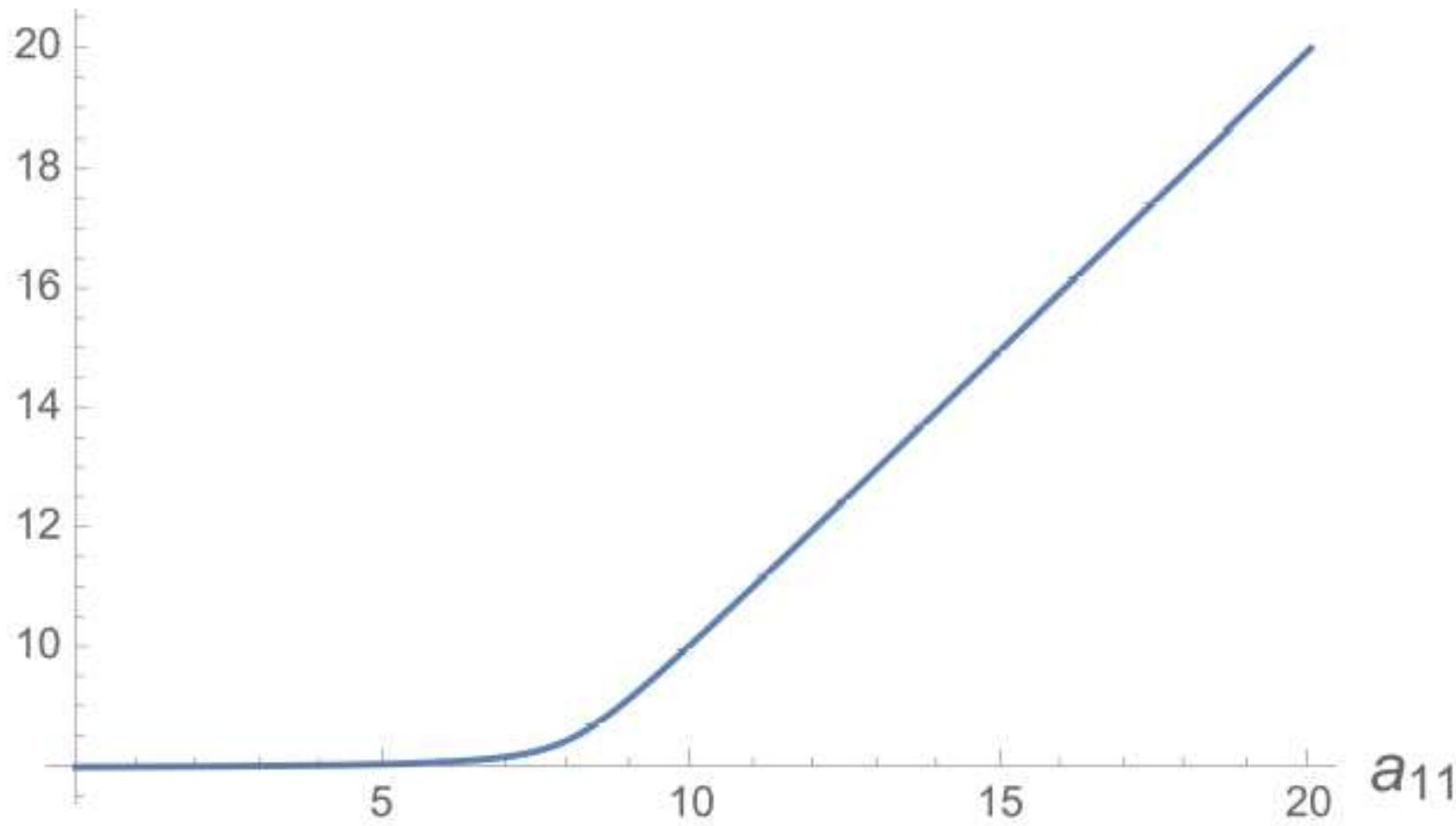

\section{Figure 7}

The largest eigenvalue of $A=($ aij $)$ as function of $a 11$, with $a 12=a 21=0.5$ and $a 22=8$.

$\mathrm{I}(\mathrm{t})$

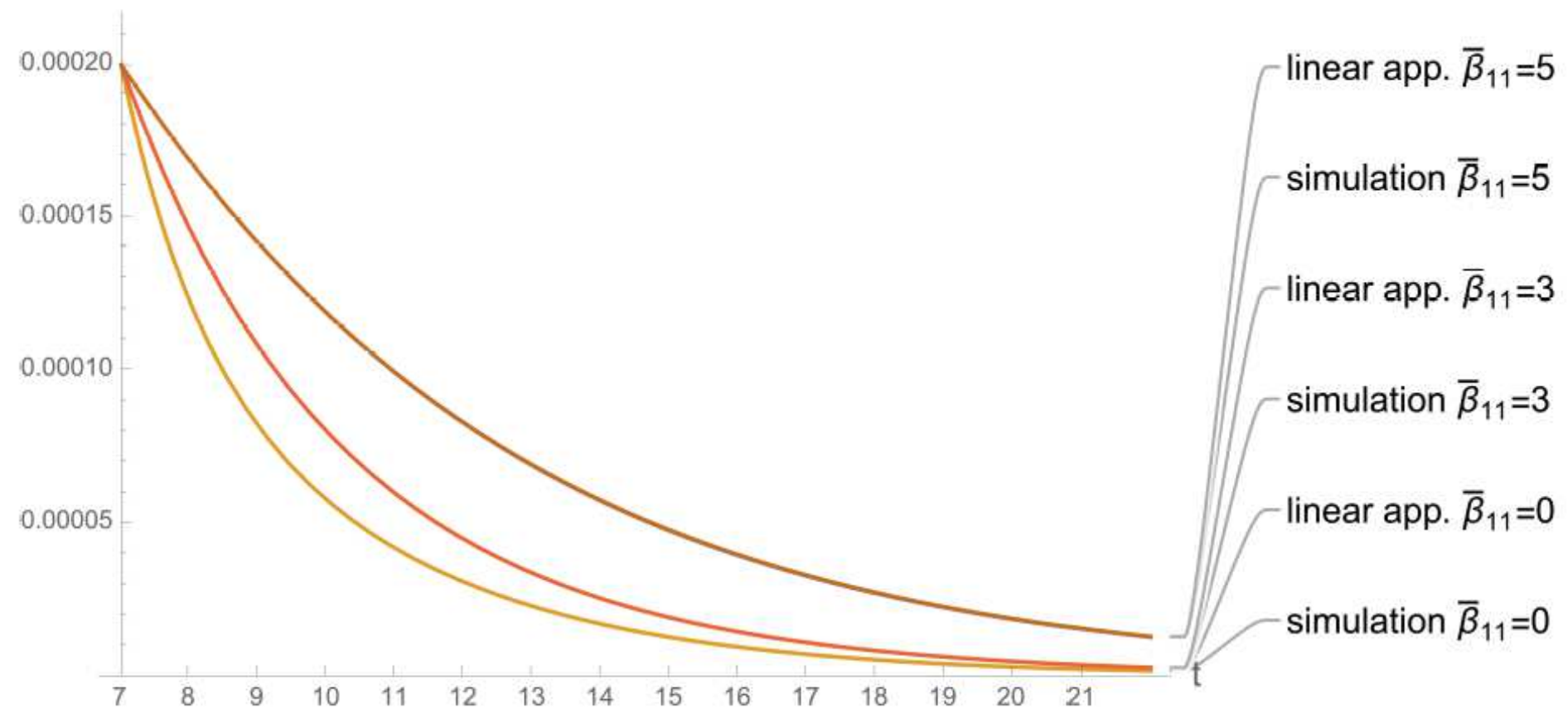

Figure 8 
Effectiveness of the linear approximation of the SIR model for lockdown; the figure shows the total active cases numerically simulated with realistic parameters and varying $₫ \beta 11$ : in each test, simulations from the differential system and from its linear approximation are indistinguishable.

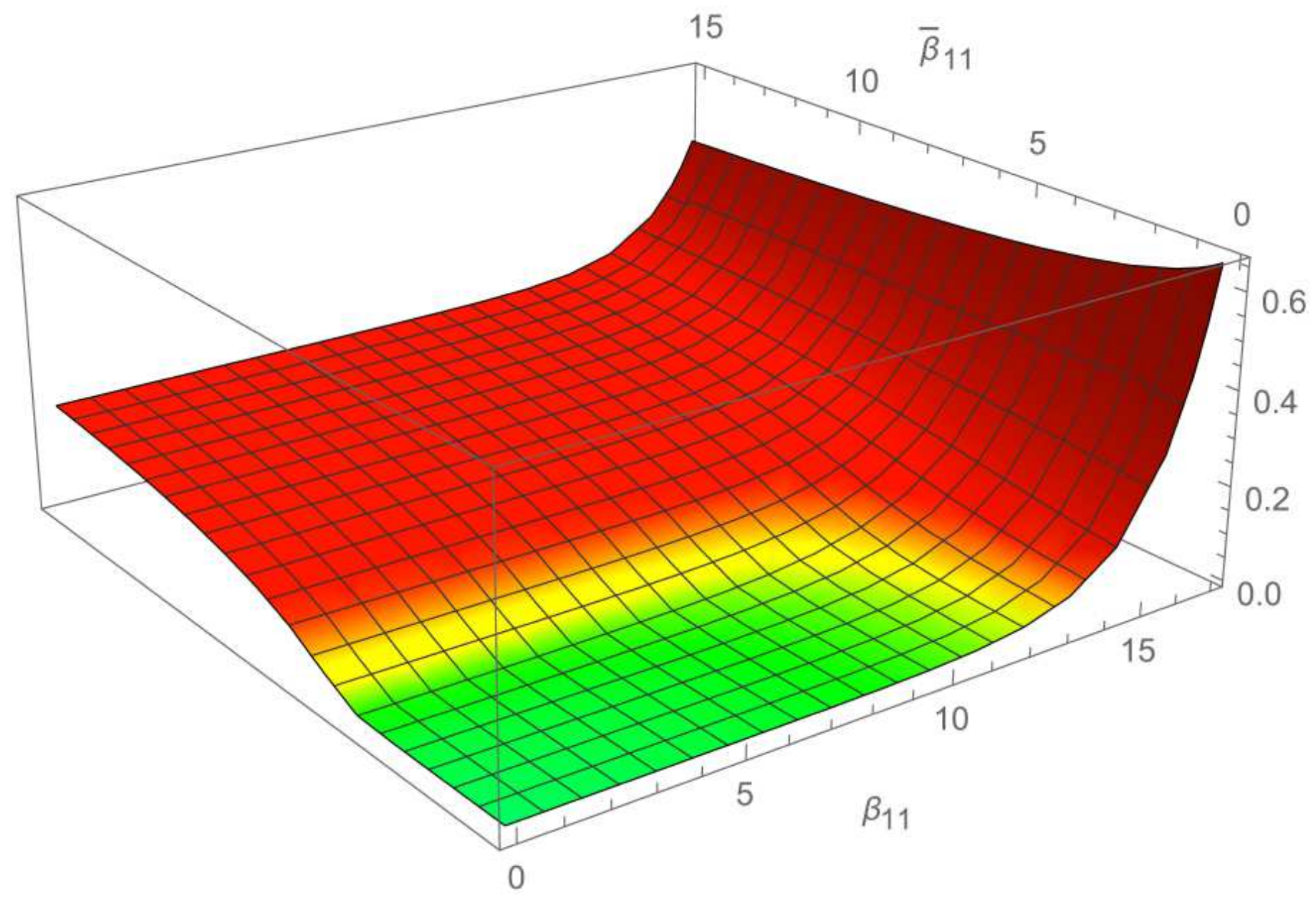

Figure 9

Total number of cases at resolution of outbreak for varying values of $\beta 11$ and $\otimes \beta 11$. 


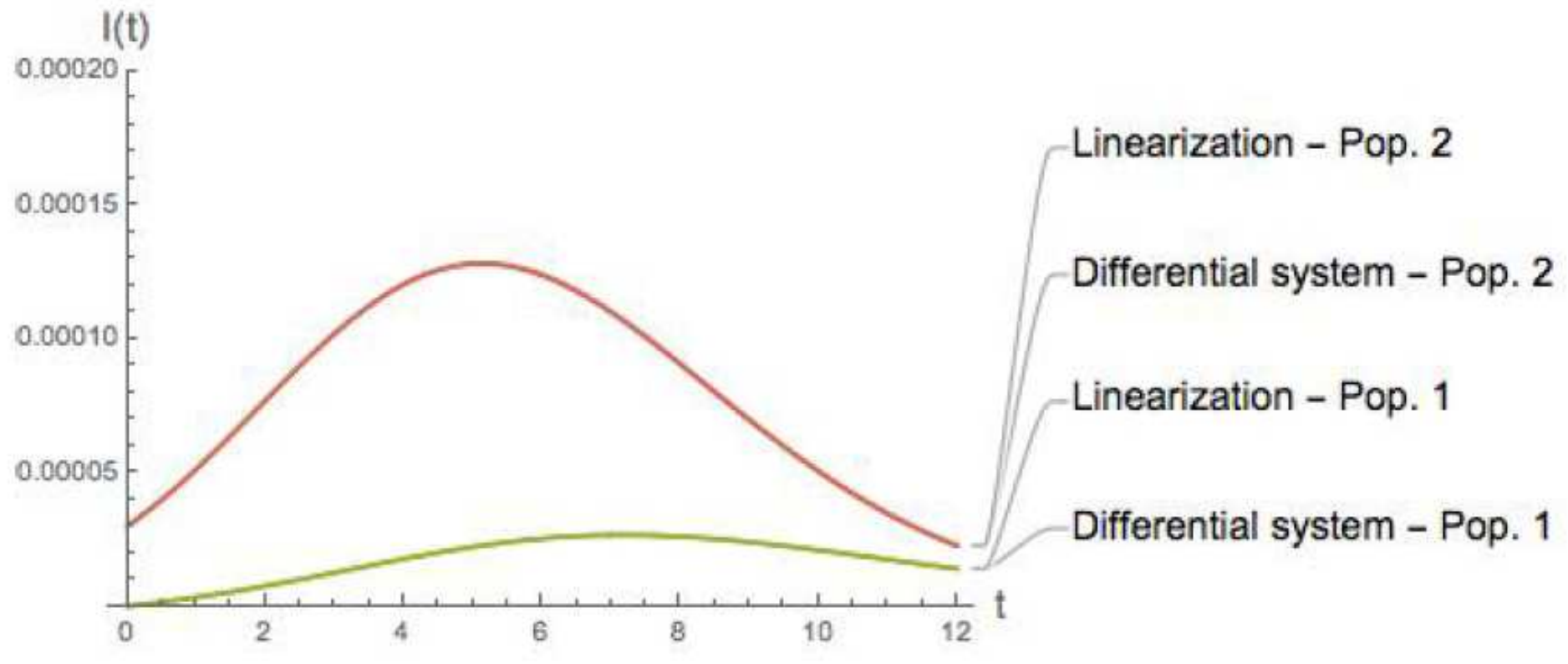

Figure 10

Effectiveness of the linear approximation of the SIR model with vaccination; the figure shows the total active cases numerically simulated with realistic parameters, $₫ \beta 11=3, \rrbracket \beta 22=2$ : simulations from the differential system and from its linear approximation are indistinguishable.

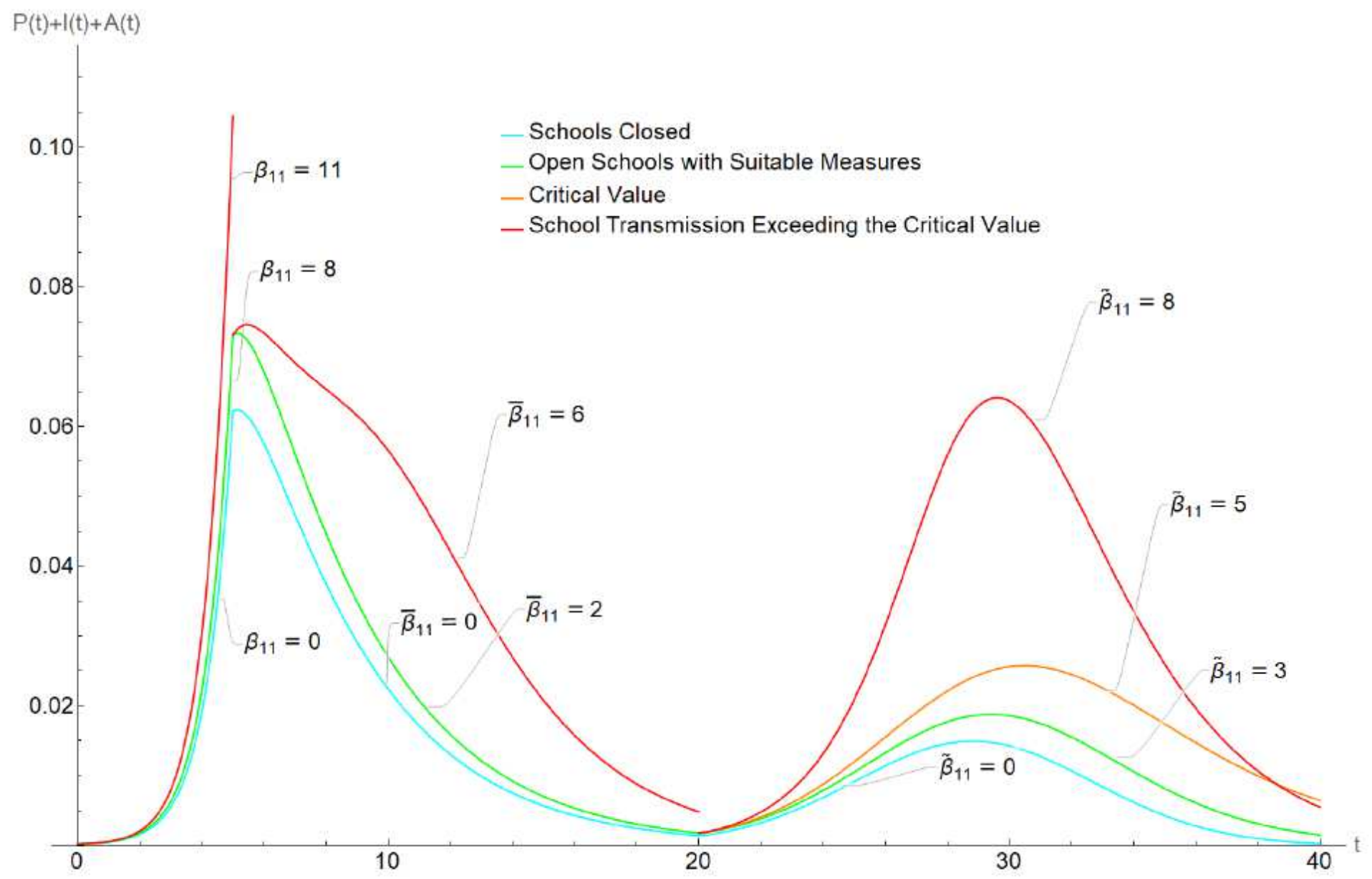




\section{Figure 11}

Daily cases for the three scenarios of outbreak, lockdown, and vaccination in an SPIAR model. In each case, there is critical values for the in-school transmission rate $\beta 11$, as for SIR model. Cyan curve is with closed schools, green for safe opening, orange for critical values, red for values above criticality. 